\title{
Kimberley marine biota. Historical data: marine plants
}

\author{
John M. Huisman ${ }^{1,2^{*}}$ and Alison Sampey ${ }^{3}$ \\ 1 Western Australian Herbarium, Science Division, Department of Parks and Wildlife, Locked Bag 104, \\ Bentley DC, Western Australian 6983, Australia. \\ 2 School of Veterinary and Life Sciences, Murdoch University, Murdoch, Western Australian 6150, \\ Australia. \\ ${ }^{3}$ Department of Aquatic Zoology, Western Australian Museum, Locked Bag 49, Welshpool DC, \\ Western Australian 6986, Australia.
}

*Email: john.huisman@dpaw.wa.gov.au

\begin{abstract}
Here, we document 308 species of marine flora from the Kimberley region of Western Australia based on collections held in the Western Australian Herbarium and on reports on marine biodiversity surveys to the region. Included are 12 species of seagrasses, 18 species of mangrove and 278 species of marine algae. Seagrasses and mangroves in the region have been comparatively well surveyed and their taxonomy is stable, so it is unlikely that further species will be recorded. However, the marine algae have been collected and documented only more recently and it is estimated that further surveys will increase the number of recorded species to over 400. The bulk of the marine flora comprised widespread Indo-West Pacific species, but there were also many endemic species with more endemics reported from the inshore areas than the offshore atolls. This number also will increase with the description of new species from the region. Collecting across the region has been highly variable due to the remote location, logistical difficulties and resource limitations. Consequently, large portions of the region remain inadequately sampled, and within the Western Australian Herbarium collections much material awaits identification.
\end{abstract}

KEYWORDS: natural history collections, species inventory, Kimberley Marine Bioregion, biodiversity, NW Australia, baseline, macroalgae, seagrass, mangrove

\section{INTRODUCTION}

Baseline marine biodiversity information to inform conservation and environmental management decisions (Pyke and Ehrlich 2010) is particularly important in the Kimberley region of Australia as it is not only an area of great conservation value, but also of extensive developments in oil and gas, and fishing and aquaculture (Department of Environment and Conservation 2009).

Australian natural science institutions, including the Western Australian Museum (WAM) have undertaken marine biodiversity surveys of the species in the Kimberley Project Area (see Methods). However, much of the data are not readily accessible, being either unpublished or in specialist taxonomic literature, so WAM initiated a data compilation of all marine organisms that will assess the state of marine biodiversity knowledge.

\section{MARINE FLORA}

This contribution deals with historical collections of the marine benthic flora of the Kimberley Project Area. This group includes all attached photosynthetic organisms ('plants' in the broad sense) and is comprised of submerged seagrasses and macroalgae, and the intertidal mangroves. Only partially included in this study are photosynthetic bacteria (cyanobacteria or bluegreen algae), as the group is in dire need of revision and most records are unreliable.

As photosynthetic primary producers, the marine benthic flora, along with the phytoplankton and symbiotic zooxanthellae, form the foundation for the complex food web that occurs in all marine ecosystems. In addition to this essential role, the benthic flora provide habitat for numerous faunal and floral organisms, give structural support by consolidating reefs and contribute substantially 
to the sediment budget. Therefore they form a significant component of all marine ecosystems. As sessile photosynthetic organisms they are also susceptible to environmental perturbation, particularly changes in water quality such as increased turbidity.

The raison d'être for collecting and studying marine plants is essentially the same as that for all biodiversity investigations: the accumulation of knowledge. Very little is known about the diversity and ranges of these organisms, particularly in remote areas as defined by the Kimberley Project Area. Elsewhere marine plants are economically important and some are used as food (e.g. Porphyra or 'nori'), as food additives, or in industrial processes. Several marine plants are also serious pests and have been introduced, accidentally or intentionally, to regions outside their native ranges. Once established, these pests are virtually impossible to eradicate so it is important the native flora are well documented and monitored for possible pest incursions (Huisman et al. 2008). Some species (e.g. Undaria pinnatifida or 'wakame') are not only economically important as a food source, but also serious pests outside their native ranges.

\section{HISTORY OF MARINE FLORAL COLLECTING IN THE KIMBERLEY PROJECT AREA}

Published historical records of marine plants in the Project Area are very scant. The voyages of discovery undertaken by British and French expeditions in the 1800 s either rarely collected marine plants or, if they were collected, the specimens were not included in published reports. So although limited historical collections may be housed in overseas herbaria, these are not addressed in the present publication.

WAM and the Western Australian Herbarium (official abbreviation PERTH) have undertaken a number of surveys of marine flora in the Project Area since the 1970s (Table 1). Surveys of the mangrove flora were undertaken in conjunction with bird surveys at 83 sites between Cambridge Gulf in the Kimberley and Shark Bay in the Gascoyne region of Western Australia between 1973 and 1982, with 14 species of mangroves collected from 25 locations in the Kimberley (Johnstone 1990). In 1995, 12 species of mangroves were recorded at sites along the southern Kimberley coast (Hanley 1995) and 14 species were recorded in the eastern Kimberley (Saenger 1996).

Five species of seagrasses were collected during the 1991 survey of Kimberley islands and reefs (Walker 1992). Seagrasses were also surveyed along the southern Kimberley coast by Walker in 1994, where she recorded eight species of seagrasses and commented on the extensive seagrass beds at Sunday and Tallon Islands being the largest in the Kimberley (Walker 1995). Only three species of seagrasses were recorded during the 1995 survey to the eastern Kimberley (Walker 1996) and along the central Kimberley coast in 1996 (Walker 1997). As there was no overlap in the species a total of six species were recorded from these two expeditions. Huisman et al. (2009) recorded three seagrasses from the shelf edge atolls, but an earlier record of Thalassodendron ciliatum from Scott Reef by Walker and Prince (1987) brings the atoll total to four. Pike and Leach (1997) and Brown and Skewes (2005) recorded five species of seagrasses at Ashmore Reef. In an earlier publication, Walker and Prince (1987) summarised the seagrass diversity in north-western Australia, recording a total of 12 species from the Kimberley, and none of the recent surveys have increased that number.

In a compendium of records of marine algae from northern Australia, Lewis (1984, 1985, 1987) included some 800 species, only nine of which were attributed to the north Western Australian coast, and of that small number none were from the Kimberley Project Area. Thus, until relatively recently, the marine algal flora of the Kimberley region was virtually unknown. Subsequent collections and publications have increased the number of species known (e.g. Phillips et al. 1993; King and Puttock 1994; Huisman 2002, 2006; Leliaert et al. 2007; Leliaert et al. 2008; Huisman et al. 2009), but the greater proportion of recent collections remains unpublished. Expeditions to the southern (1994), eastern (1995) and central (1996) Kimberley recorded 72, 90 and 70 species of macroalgae, respectively (Walker 1997). More recently, 117 species of macroalgae were recorded from the shelf edge atolls (Huisman et al. 2009), and a survey of the benthic flora of Gourdon Bay and the Dampier Peninsula recorded one seagrass and 43 algal species (Table 1), including one possible new species and two new records of algae for Western Australia (Keesing et al. 2011).

\section{AIM}

To synthesise records of shallow water $(<30 \mathrm{~m})$ marine flora (macroalgae, seagrass and mangrove) species in the Kimberley region of Australia that are verified by specimens lodged in herbarium collections (1880s-2009) and to provide commentary on diversity trends, taxonomic and collection gaps of the flora in the region.

\section{METHODS}

The Project Area encompasses an area west and north of the Kimberley coast (south of Broome to the Western Australia-Northern Territory border) extending beyond the $1000 \mathrm{~m}$ bathymetric contour, with the coastline forming a natural inshore boundary, as shown in Figure 1 (see Sampey et al. 2014 for a full description of the study area). 
TABLE $1 \quad$ Number of species of marine flora taxa groups sampled during main survey expeditions in the region and presented as reports. Number in parentheses is the number of locations. * Total number from report, but data not included in our species list as the tables were incomplete in the report.

\begin{tabular}{|c|c|c|c|c|c|c|c|}
\hline Survey year & $\begin{array}{l}\text { Blue-green } \\
\text { algae }\end{array}$ & $\begin{array}{l}\text { Red } \\
\text { algae }\end{array}$ & $\begin{array}{l}\text { Brown } \\
\text { algae }\end{array}$ & $\begin{array}{l}\text { Green } \\
\text { algae }\end{array}$ & Seagrasses & Mangroves & Reference \\
\hline 1973-1982 & & & & & & $14(25)$ & Johnstone 1990 \\
\hline 1991 & & & & & $5(5)$ & & Walker 1992 \\
\hline 1994 & 3 genera* & $28^{*}$ & $21^{*}$ & $23^{*}$ & $8(14)$ & $13(8)$ & Hanley 1995; Walker 1995 \\
\hline 1995 & & $37(11)$ & $18(7)$ & $35(23)$ & $3(3)$ & $11(11)$ & Saenger 1996; Walker 1996 \\
\hline 1996 & & $34(15)$ & $14(9)$ & $22(19)$ & $3(7)$ & & Walker 1997 \\
\hline 1997-2001 & & & & & $5(1)$ & & Brown and Skewes 2005 \\
\hline 2006 & $3(2)$ & $65(3)$ & $9(3)$ & $40(12)$ & $4(3)$ & & Huisman et al. 2009 \\
\hline 2008 & & $28(4)$ & $7(3)$ & $8(3)$ & $1(2)$ & & Keesing et al. 2011 \\
\hline
\end{tabular}

The marine flora documented here essentially forms an artificial assemblage united by habitat rather than phylogeny. Included are 'higher' plants (seagrasses and mangroves, the latter also an artificial assemblage), algae (Rhodophyta, Phaeophyceae, Ulvophyceae) and blue-green algae (Cyanobacteria).

The methodology follows that outlined by Sampey et al. (2014). Marine flora data were sourced from the PERTH database (data extracted February 2010) and the species lists presented in seven reports (Table 1; Johnstone 1990; Walker 1992, 1995, 1996, 1997; Hanley 1995; Saenger 1996; Brown and Skewes 2005; Huisman et al. 2009). Species lists for seagrasses and macroalgae in the 1994 survey report (Walker 1995) were incomplete and only 4 of the 22 stations were printed, but there were specimens lodged in PERTH that are included in our dataset. The marine flora recorded by the 2008 CSIRO survey along the Dampier Peninsula and Gourdon Bay (Keesing et al. 2011) were made available only late in the preparation of this dataset, so we have not included the species or locations in this present dataset. The specimens from that collection will be incorporated into the PERTH collection and database when resources are available.

Species names represent a hypothesis that is subject to change as new information (morphology, genetic, behaviour, distribution ranges) is discovered (Gaston and Mound 1993). The species names and taxonomic placement of the records in the dataset were checked in an endeavour to present the currently accepted name and resolve synonymies and old combinations, but the specimens were not re-examined for this study (for full details see Sampey et al. 2014). Species names were checked for current taxonomic placement and validity using a variety of online resources (ABRS 2011; Cowan 2011; Guiry and Guiry 2011; The International Plant Names Index 2011; Western Australian Herbarium 2011) and relevant recent publications.

\section{SPATIAL INFORMATION, COLLECTION DETAILS AND MAPPING}

As described by Sampey et al. (2014) data from all sources were collated into a single database. Location and collection details were checked and verified. The location of the specimen records were visualised using ARCGIS v9, ArcMap v9.3 and outliers in particular were examined and the latitude and longitude corrected or the record excluded as appropriate. Maps of species richness and sampling effort were generated for each main location. Since species richness patterns are highly dependent on sampling effort, we calculated the number of collecting events at a location to provide an indication of relative sampling effort. A collecting event was defined by the season and year of collecting and the full list of locations, latitude and longitude and other relevant collection information is provided in Table 2 of Sampey et al. (2014). 


\section{BIOGEOGRAPHIC AND HABITAT CODING}

Species were coded for their known habitat and biogeographic range to provided extra information for researchers and managers.

Biogeography codes used were:

- Western Australian endemic (WA). Currently only known from Western Australian waters, often from the type locality only; may eventually prove to be a northern Australian endemic with more collecting effort.

- Northern Australian endemic (NA). Found throughout tropical Australian waters.

- Australian endemic (A). Found throughout tropical and temperate Australian waters.

- Indo-Australian (IA). Found throughout Australian and Indonesian waters, may extend to the Philippines and for some species Japan.

- Indian Ocean (IO). Restricted to the Indian Ocean.

- Indo-West Pacific (IWP). Found throughout the Red Sea, Indian and Western Pacific Oceans.

- Indo-Pacific (IP). Found throughout the Red Sea, Indian Ocean and throughout the Pacific Ocean.

- Tropicopolitan (T). Found throughout all tropical oceans.

- Circum-global (C). Found throughout all oceans in both tropical and temperate waters.

A species may be restricted to certain habitat types within its distribution range. The species in this dataset were coded for their preferred habitat, if known, as follows:
- Intertidal (superscript i). Species is found in the intertidal zone.

- Subtidal (superscript s). Species is found in the subtidal zone.

- Hard Substrate (H). Species is found associated with hard substrates (e.g. rock, coral, rubble).

- Soft Substrate (S). Species is found associated with soft substrates (e.g. sand, mud).

- Epiphytic (EP). Always found in an external association with a particular species of marine plant.

- Endophytic (EnP). Always found in an internal association with a particular species of marine plant, i.e. living within a marine plant.

- Unknown (U).

Combinations of these codes were used as appropriate.

\section{RESULTS}

\section{NUMBER OF SPECIMENS IN COLLECTIONS}

A total of 1431 registered specimen lots of marine flora were retained in this dataset (Table 2). These included green algae $(353,24.5 \%)$, blue-green algae $(6,0.5 \%)$, brown algae $(284,20 \%)$, red algae $(502$, $35 \%)$, seagrasses $(107,7.5 \%)$, and mangroves (179, $12.5 \%$ ) (Table 2). Many lots were excluded from the present dataset either because they were freshwater species collected in streams, waterholes or rivers in the Kimberley and therefore not relevant to our project, or because of incomplete identification (1,342 lots, Table 2).

TABLE 2

Number of registered specimen lots of Kimberley Project Area marine flora housed in the PERTH collections. Included are those lots that were identified to species or able to be distinguished as a separate species and excluded are those lots that were incompletely identified or from freshwater locations.

\begin{tabular}{llll} 
Division & Common name & Included & Excluded \\
\hline Not specified & Algae & - & 201 \\
Cyanophyta & Blue-Green Algae & 6 & 6 \\
Rhodophyta & Red Algae & 502 & 365 \\
Heterokontophyta & Brown Algae & 284 & 309 \\
Chlorophyta & Green Algae & 353 & 236 \\
Magnoliophyta: Liliopsida & Seagrasses & 107 & 212 \\
Magnoliophyta: Magnoliopsida & Mangroves & 179 & 13 \\
Total & & $\mathbf{1 4 3 1}$ & $\mathbf{1 3 4 2}$ \\
\hline
\end{tabular}


The oldest specimen records of each marine flora taxa in the region that were present as specimens at the PERTH were the grey mangrove, Avicennia marina collected from Roebuck Bay in 1889, the seagrass Thalassia hemprichii from Cartier Island in 1977 and the algae Halimeda opuntia and Hypnea spinella collected from the fringing reef at Cartier Island by K.F. Kenneally in 1977.

With interest in the region's flora only relatively recent, so far very few new marine taxa have been based on Kimberley type specimens, but this will change with the publication of a north-western Australian algal flora currently in preparation and including descriptions of at least 50 new species (Huisman, in preparation). A Russian expedition in 1978 resulted in the description of a new genus and species of coralline red algae, Rhizolamellia collum from Scott Reef (Shevejko 1982), and the species Liagora walkerae was described from Cassini Island by Huisman (2002).

\section{SPECIES RICHNESS AND COLLECTING EFFORT}

A total of 308 species of marine flora were recorded in our dataset for the Project Area. These included green algae ( 88 species, $28.5 \%$ ); blue-green algae, (6 species, $2 \%$ ), brown algae, (39 species, 12.5\%), red algae (145 species, $47 \%$ ); seagrasses (12 species, 4\%); and mangroves (18 species, 6\%); Table 3 ). These proportions are relatively typical, with the red algae the most speciose, followed by the green and brown algae. No mangroves have been recorded from the offshore atolls in the Project Area as suitable habitat does not occur.

TABLE 3 Species of marine flora recorded from the Project Area.

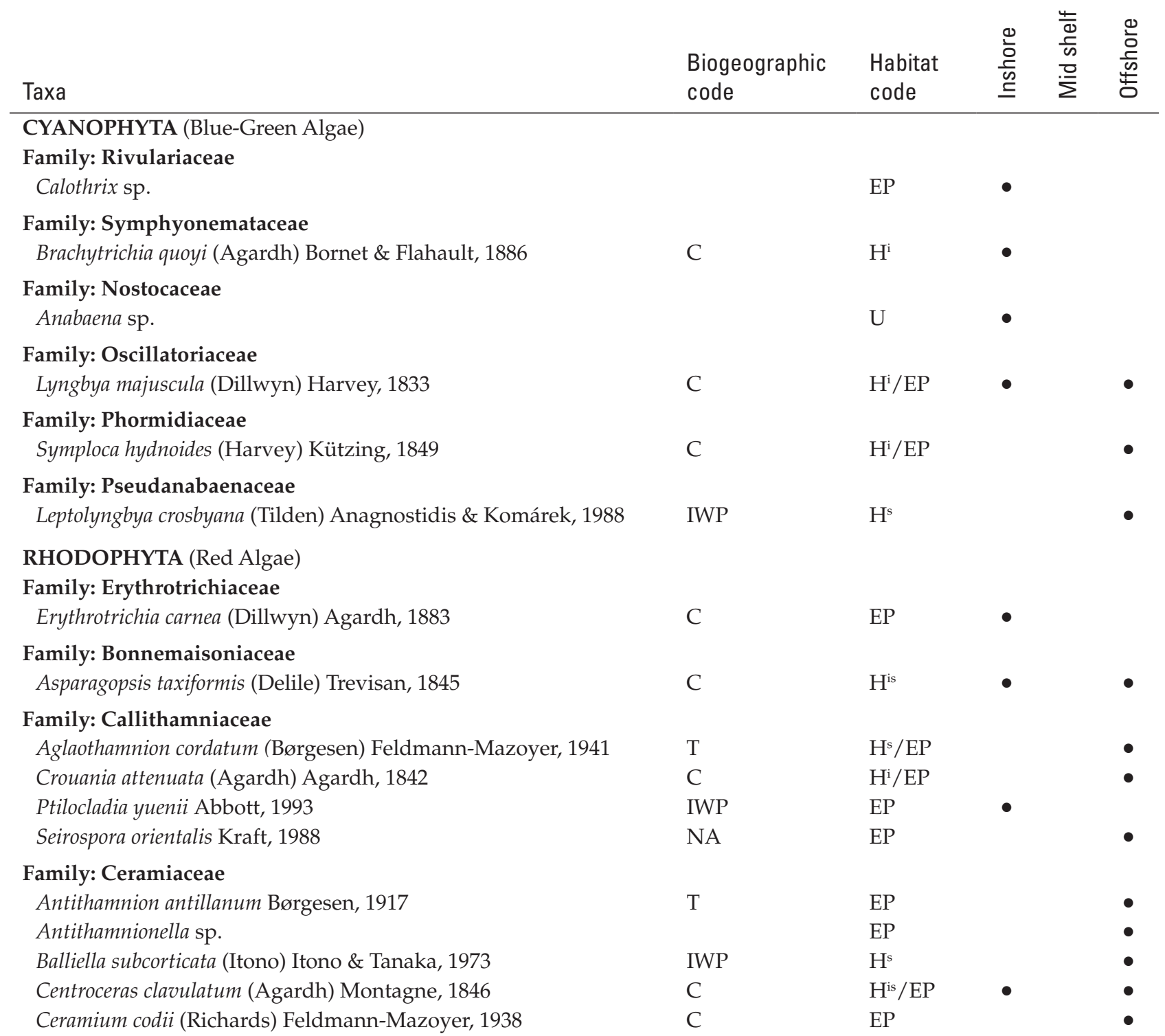




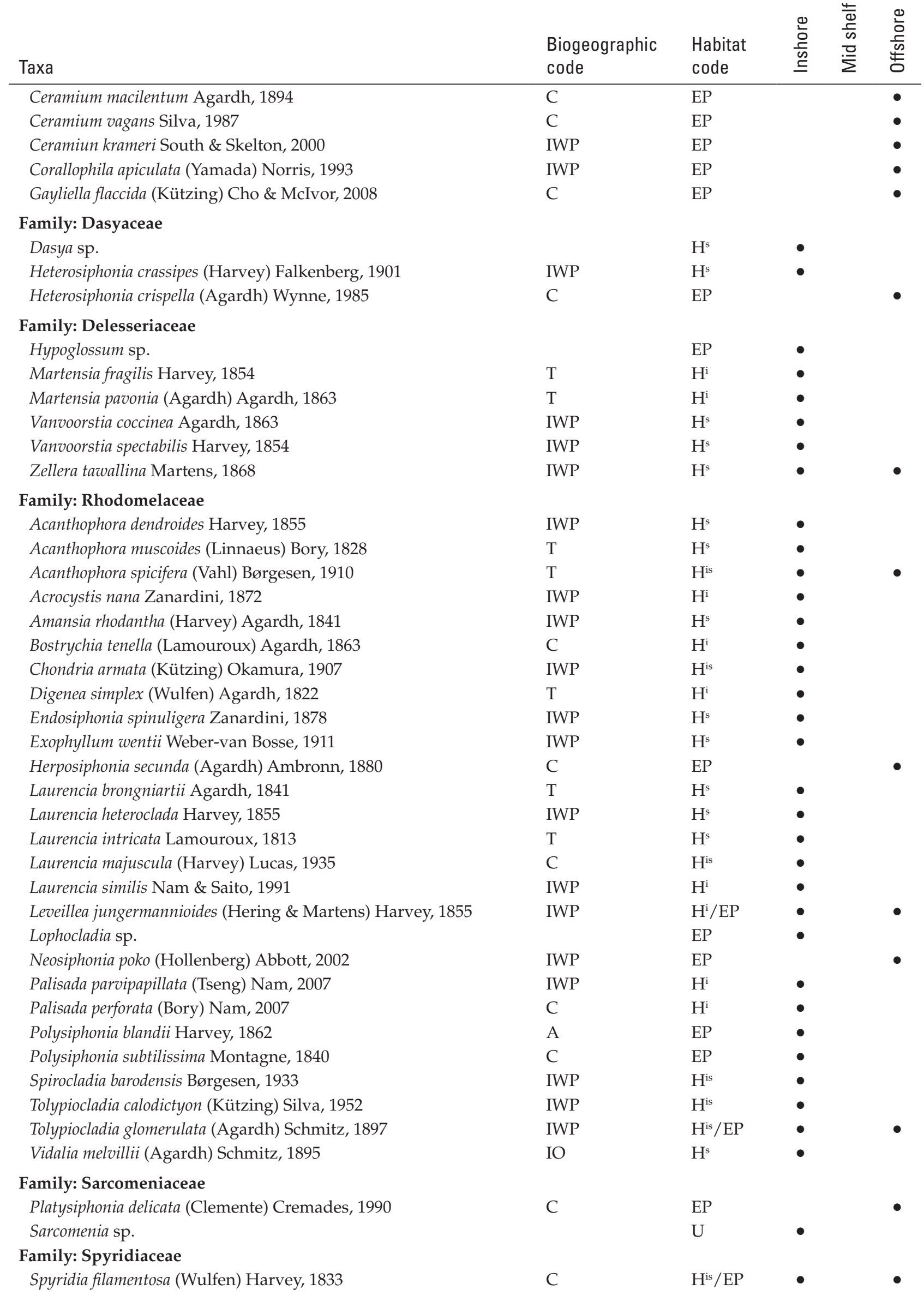




\begin{tabular}{|c|c|c|c|c|}
\hline Taxa & $\begin{array}{l}\text { Biogeographic } \\
\text { code }\end{array}$ & $\begin{array}{l}\text { Habitat } \\
\text { code }\end{array}$ & 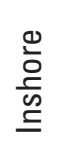 & $\begin{array}{l}\frac{\omega}{\omega} \\
\frac{1}{\omega} \\
\frac{D}{\Sigma}\end{array}$ \\
\hline \multicolumn{5}{|l|}{ Family: Wrangeliaceae } \\
\hline Anotrichium tenue (Agardh) Nägeli, 1862 & $\mathrm{C}$ & $\mathrm{H}^{\mathrm{is}} / \mathrm{EP}$ & - & \\
\hline Griffithsia heteromorpha Kützing, 1863 & $\mathrm{~T}$ & EP & & \\
\hline Haloplegma duperreyi Montagne, 1842 & $\mathrm{~T}$ & $\mathrm{H}^{\text {is }}$ & & \\
\hline Haloplegma preissii (Harvey) Montagne, 1845 & A & $\mathrm{H}^{\text {is }}$ & - & \\
\hline Spongoclonium caribaeum (Børgesen) Wynne, 2005 & C & $\mathrm{H}^{\mathrm{is}} / \mathrm{EP}$ & - & \\
\hline Tiffaniella cymodoceae (Børgesen) Gordon-Mills, 1972 & IO & $\mathrm{EP}$ & & \\
\hline Wrangelia elegantissima Norris, 1994 & IWP & $\mathrm{H}^{\mathrm{i}}$ & $\bullet$ & \\
\hline \multicolumn{5}{|l|}{ Family: Corallinaceae } \\
\hline Amphiroa beauvoisii Lamouroux, 1816 & $\mathrm{C}$ & $\mathrm{H}^{\text {is }}$ & $\bullet$ & \\
\hline Amphiroa foliacea Lamouroux, 1824 & $\mathrm{~T}$ & $\mathrm{H}^{\text {is }}$ & $\bullet$ & \\
\hline Amphiroa fragilissima (Linnaeus) Lamouroux, 1816 & $\mathrm{~T}$ & $\mathrm{H}^{\text {is }}$ & $\bullet$ & \\
\hline Amphiroa gracilis Harvey, 1855 & IWP & $\mathrm{H}^{\mathrm{s}}$ & - & \\
\hline Hydrolithon farinosum (Lamouroux) Penrose \& Chamberlain, 1993 & $\mathrm{C}$ & $\mathrm{H}^{\mathrm{is}} / \mathrm{EP}$ & - & \\
\hline Hydrolithon gardineri (Foslie) Verheij \& Prud'homme van Reine, 1993 & IP & $\mathrm{H}^{\text {is }}$ & & \\
\hline Hydrolithon munitum (Foslie \& Howe) Penrose, 1996 & $\mathrm{~T}$ & $\mathrm{H}^{\text {is }}$ & & \\
\hline Hydrolithon onkodes (Heydrich) Penrose \& Woelkerling, 1992 & $\mathrm{~T}$ & $\mathrm{H}^{\text {is }}$ & & \\
\hline Hydrolithon samoense (Foslie) Keats \& Chamberlain, 1994 & C & $\mathrm{H}^{\text {is }}$ & & \\
\hline Jania adhaerens Lamouroux, 1816 & C & $\mathrm{EP}$ & - & \\
\hline Jania micrarthrodia Lamouroux, 1816 & C & $\mathrm{EP}$ & - & \\
\hline Lithophyllum insipidum Adey, Townsend \& Boykins, 1982 & IWP & $\mathrm{H}^{\text {is }}$ & - & \\
\hline Lithophyllum kotschyanum Unger, 1858 & $\mathrm{~T}$ & $\mathrm{H}^{\text {is }}$ & - & \\
\hline Lithophyllum pygmaeum (Heydrich) Heydrich, 1897 & IWP & $\mathrm{H}^{\mathrm{is}}$ & $\bullet$ & \\
\hline Lithophyllum tamiense (Heydrich) Foslie, 1900 & IWP & $\mathrm{H}^{\mathrm{is}}$ & & \\
\hline Lithothamnion proliferum Foslie, 1904 & IWP & $\mathrm{H}^{\mathrm{s}}$ & & \\
\hline Mastophora rosea (Agardh) Setchell, 1943 & IWP & $\mathrm{H}^{\mathrm{i}}$ & $\bullet$ & \\
\hline Rhizolamellia collum Shevejko, 1982 & WA & $\mathrm{H}^{\mathrm{s}}$ & & \\
\hline \multicolumn{5}{|l|}{ Family: Gelidiaceae } \\
\hline Gelidiella acerosa (Forsskål) Feldmann \& Hamel, 1934 & $\mathrm{~T}$ & $\mathrm{H}^{\mathrm{i}}$ & - & \\
\hline Gelidium sp. & & $\mathrm{H}^{\mathrm{i}}$ & - & \\
\hline Pterocladiella caerulescens (Kützing) Santelices \& Hommersand, 1997 & $\mathrm{~T}$ & $\mathrm{H}^{\mathrm{is}}$ & $\bullet$ & \\
\hline \multicolumn{5}{|l|}{ Family: Areschougiaceae } \\
\hline Erythroclonium sp. nov. & WA & $\mathrm{H}^{\mathrm{s}}$ & $\bullet$ & \\
\hline \multicolumn{5}{|l|}{ Family: Caulacanthaceae } \\
\hline Catenella nipae Zanardini, 1872 & IWP & $\mathrm{H}^{\mathrm{i}} / \mathrm{EP}$ & $\bullet$ & \\
\hline \multicolumn{5}{|l|}{ Family: Corynocystaceae } \\
\hline Corynocystis prostrata Kraft, 1999 & IWP & $\mathrm{H}^{\mathrm{s}}$ & & \\
\hline \multicolumn{5}{|l|}{ Family: Corynomorphaceae } \\
\hline Corynomorpha prismatica (Agardh) Agardh, 1876 & IWP & $\mathrm{H} / \mathrm{S}^{\mathrm{s}}$ & $\bullet$ & \\
\hline \multicolumn{5}{|l|}{ Family: Dumontiaceae } \\
\hline Gibsmithia hawaiiensis Doty, 1963 & IWP & $\mathrm{H}^{\mathrm{s}}$ & & \\
\hline \multicolumn{5}{|l|}{ Family: Hypneaceae } \\
\hline Hypnea cornuta (Kützing) Agardh, 1851 & $\mathrm{C}$ & $\mathrm{H}^{\mathrm{is}}$ & $\bullet$ & \\
\hline Hypnea pannosa Agardh, 1847 & $\mathrm{~T}$ & $\mathrm{H}^{\mathrm{is}}$ & $\bullet$ & \\
\hline Hypnea spinella (Agardh) Kützing, 1847 & $\mathrm{C}$ & $\mathrm{H}^{\mathrm{is}}$ & $\bullet$ & \\
\hline Hypnea valentiae (Turner) Montagne, 1841 & $\mathrm{C}$ & $\mathrm{H}^{\text {is }}$ & $\bullet$ & \\
\hline \multicolumn{5}{|l|}{ Family: Kallymeniaceae } \\
\hline Kallymenia sp. & & $\mathrm{H}^{\mathrm{s}}$ & & \\
\hline
\end{tabular}




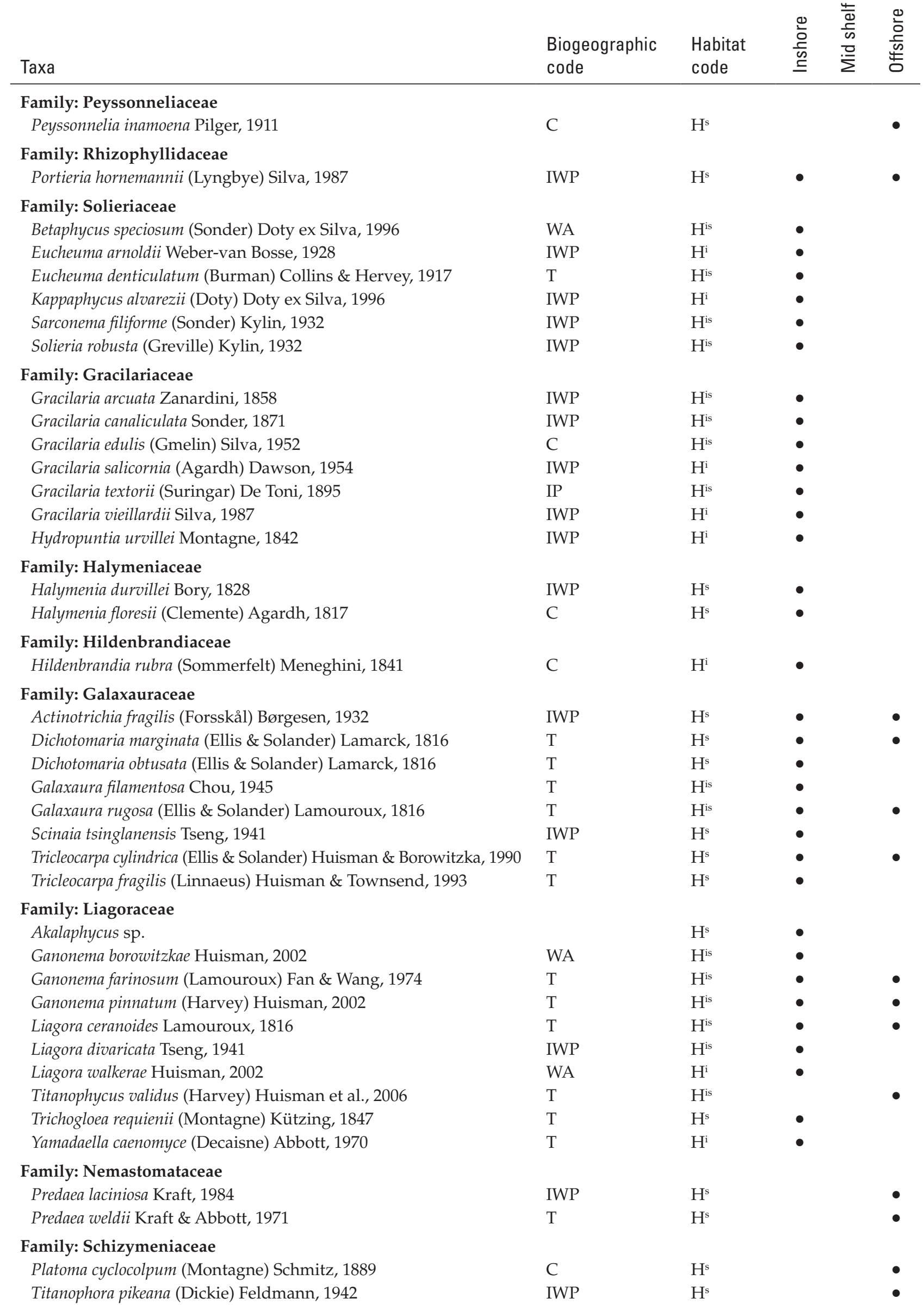




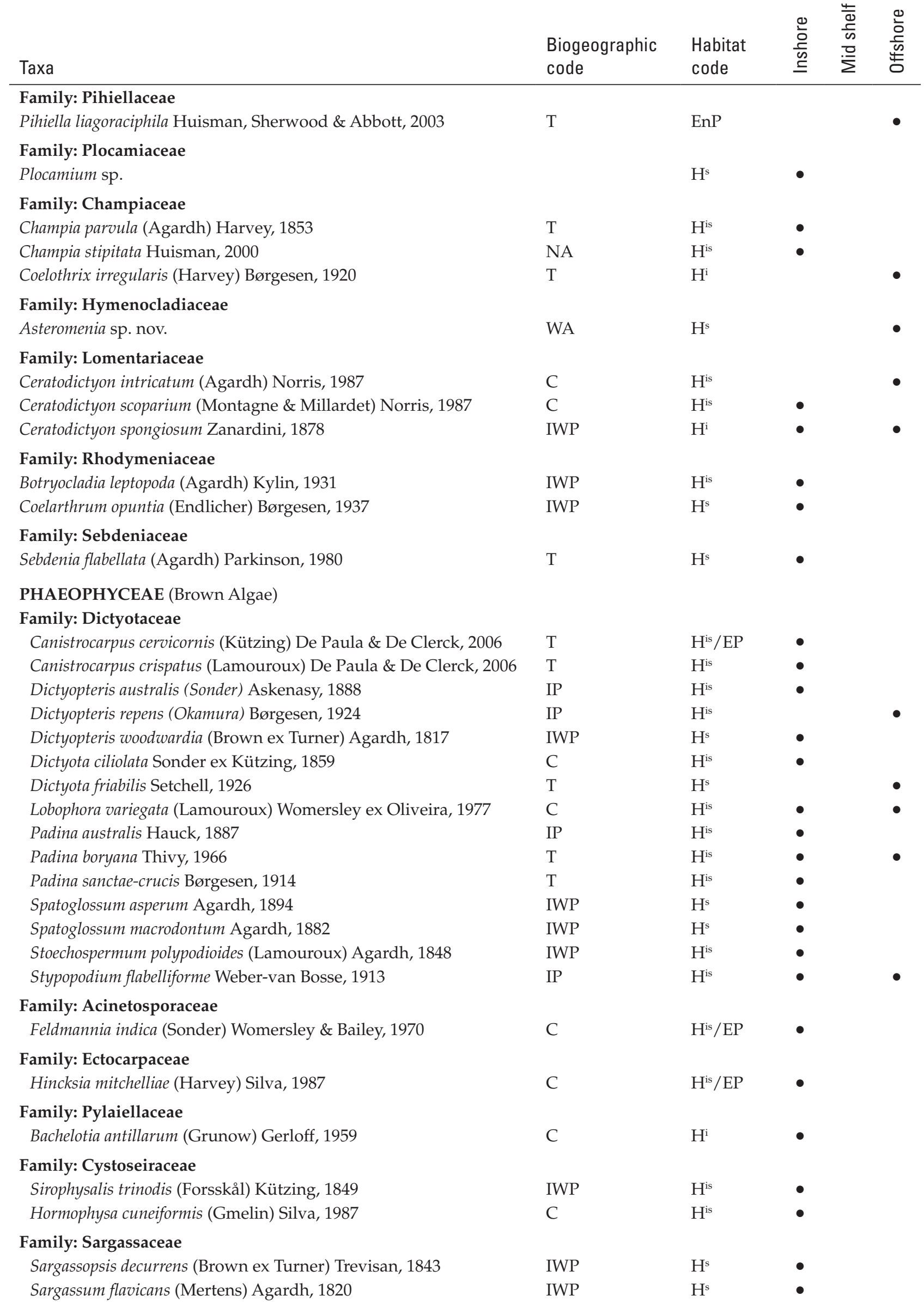




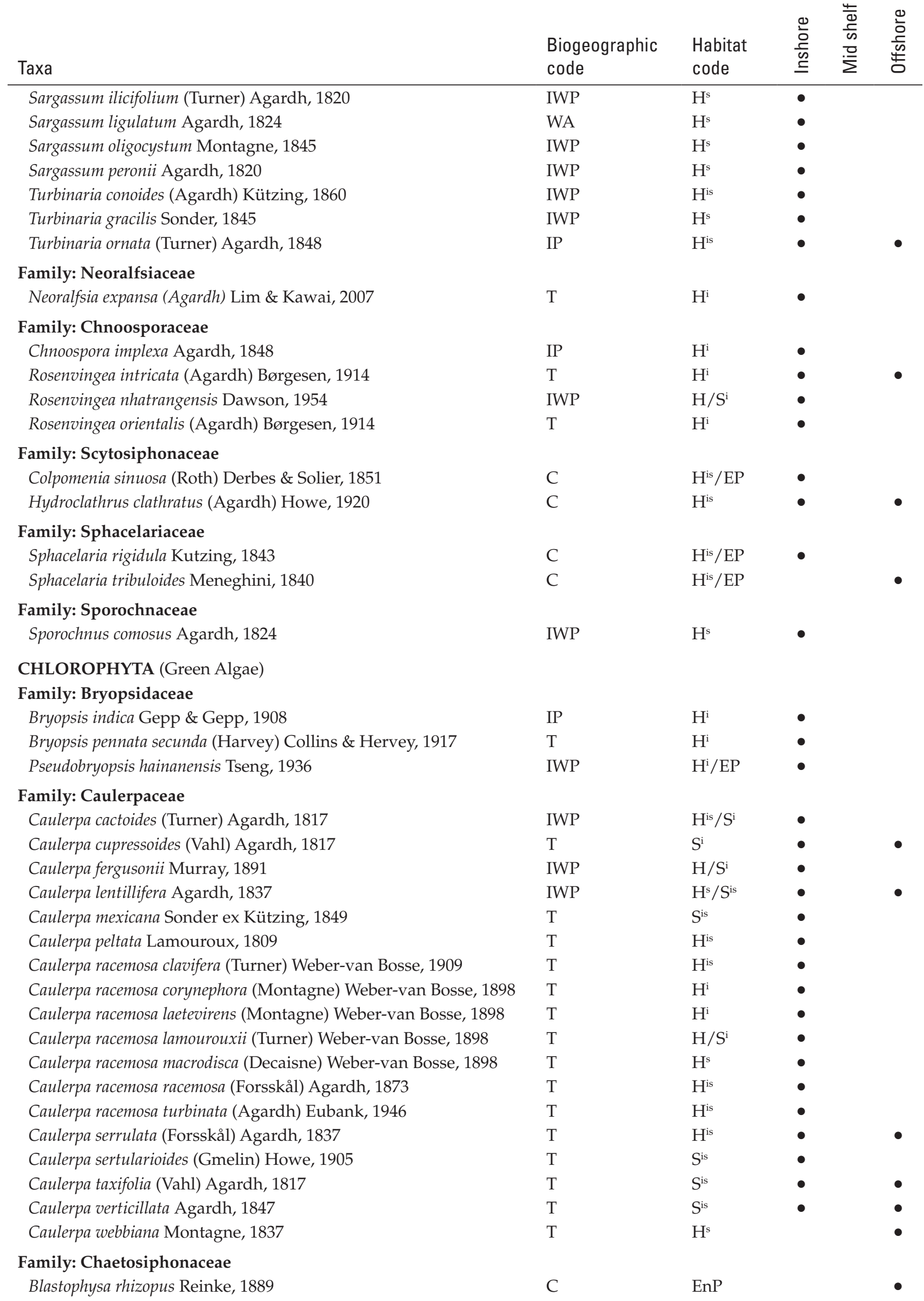




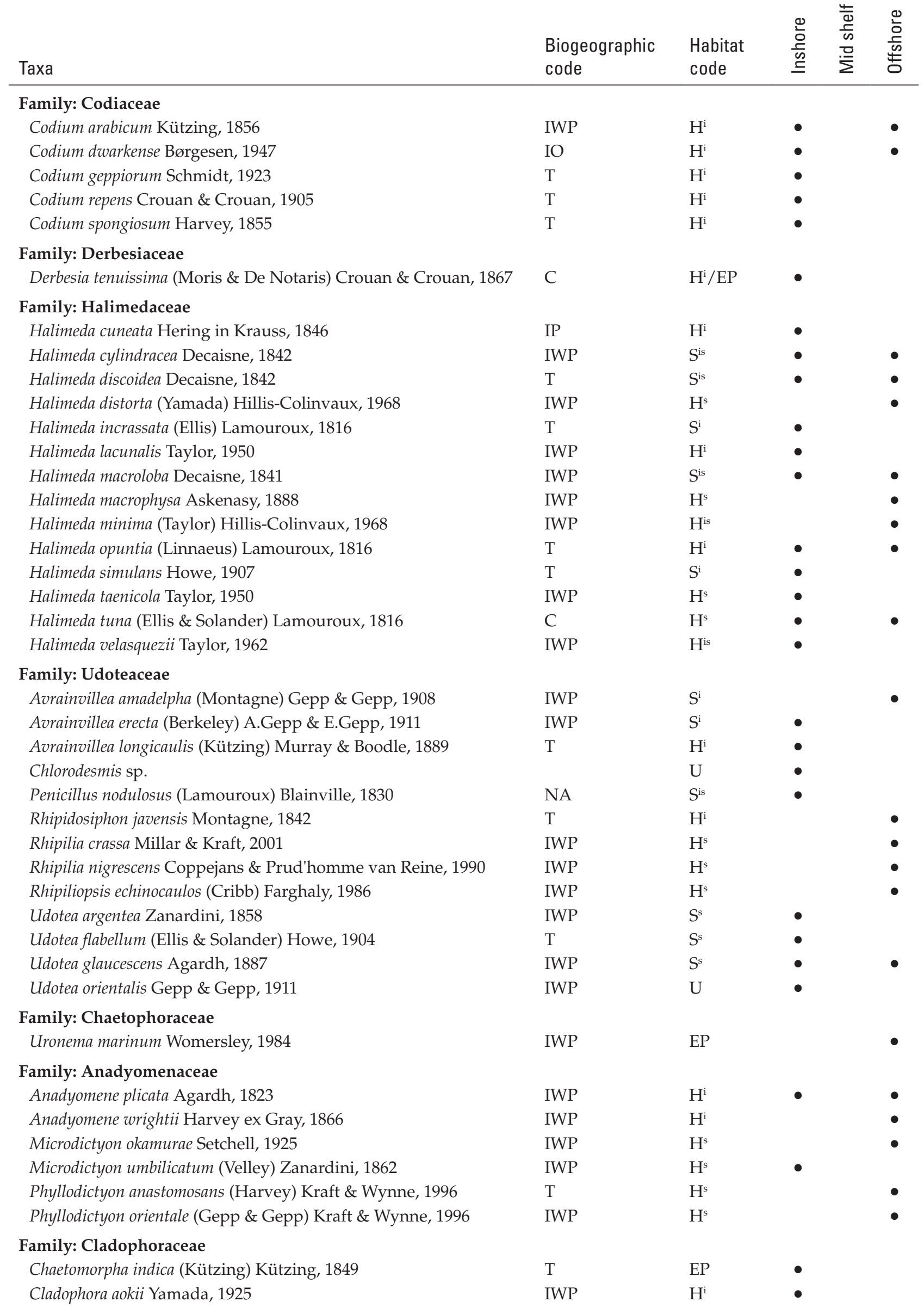




\begin{tabular}{|c|c|c|c|c|}
\hline Taxa & $\begin{array}{l}\text { Biogeographic } \\
\text { code }\end{array}$ & $\begin{array}{l}\text { Habitat } \\
\text { code }\end{array}$ & 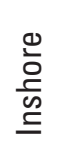 & $\begin{array}{l}\frac{4}{0} \\
\frac{D}{\omega} \\
\frac{D}{\Sigma} \\
\end{array}$ \\
\hline Cladophora catenata (Linnaeus) Kützing, 1843 & $\mathrm{~T}$ & $\mathrm{H}^{\text {is }}$ & $\bullet$ & \\
\hline Cladophora coelothrix Kützing, 1843 & $\mathrm{C}$ & $\mathrm{H}^{\text {is }}$ & & \\
\hline Cladophora herpestica (Montagne) Howe, 1914 & $\mathrm{C}$ & $\mathrm{H}^{\text {is }}$ & $\bullet$ & \\
\hline Cladophora socialis Kützing, 1849 & $\mathrm{C}$ & $\mathrm{H}^{\mathrm{i}}$ & & \\
\hline \multicolumn{5}{|l|}{ Family: Siphonocladaceae } \\
\hline Boergesenia forbesii (Harvey) Feldmann, 1938 & IWP & $\mathrm{H}^{\mathrm{i}}$ & $\bullet$ & \\
\hline Boodlea composita (Harvey) Brand, 1904 & $\mathrm{~T}$ & $\mathrm{H}^{\mathrm{i}}$ & - & - \\
\hline Boodlea vanbosseae Reinbold, 1905 & IWP & $\mathrm{H}^{\mathrm{i}}$ & & - \\
\hline Cladophoropsis sundanensis Reinbold, 1905 & $\mathrm{~T}$ & $H^{\text {is }}$ & & \\
\hline Cladophoropsis vaucheriiformis (Areschoug) Papenfuss, 1958 & IWP & $\mathrm{H}^{\mathrm{i}}$ & - & \\
\hline Dictyosphaeria cavernosa (Forsskål) Børgesen, 1932 & $\mathrm{~T}$ & $\mathrm{H}^{\text {is }}$ & $\bullet$ & \\
\hline Dictyosphaeria versluysii Weber-van Bosse, 1905 & $\mathrm{~T}$ & $\mathrm{H}^{\text {is }}$ & & \\
\hline \multicolumn{5}{|l|}{ Family: Valoniaceae } \\
\hline Valonia aegagropila Agardh, 1823 & $\mathrm{~T}$ & $\mathrm{H}^{\mathrm{s}}$ & $\bullet$ & \\
\hline Valonia fastigiata Agardh, 1887 & $\mathrm{~T}$ & $\mathrm{H}^{\mathrm{s}}$ & & \\
\hline Valonia ventricosa Agardh, 1887 & $\mathrm{~T}$ & $\mathrm{H}^{\mathrm{s}}$ & & \\
\hline Valoniopsis pachynema (Martens) Børgesen, 1934 & $\mathrm{~T}$ & $\mathrm{H}^{\mathrm{i}}$ & $\bullet$ & \\
\hline \multicolumn{5}{|l|}{ Family: Dasycladaceae } \\
\hline Bornetella oligospora Solms-Laubach, 1892 & IWP & $\mathrm{H}^{\mathrm{s}}$ & $\bullet$ & \\
\hline Bornetella sphaerica (Zanardini) Solms-Laubach, 1892 & IWP & $\mathrm{H}^{\mathrm{s}}$ & $\bullet$ & \\
\hline Neomeris bilimbata Koster, 1937 & IWP & $\mathrm{H}^{\mathrm{s}}$ & & \\
\hline Neomeris vanbosseae Howe, 1909 & $\mathrm{IP}$ & $\mathrm{H}^{\mathrm{s}}$ & $\bullet$ & \\
\hline \multicolumn{5}{|l|}{ Family: Polyphysaceae } \\
\hline Acetabularia caliculus Lamouroux in Quoi \& Gaimard, 1824 & C & $\mathrm{H}^{\mathrm{s}}$ & $\bullet$ & \\
\hline \multicolumn{5}{|l|}{ Family: Ulvaceae } \\
\hline Ulva clathrata (Roth) Agardh, 1811 & $\mathrm{C}$ & $\mathrm{H}^{\mathrm{i}}$ & $\bullet$ & \\
\hline Ulva flexuosa Wulfen, 1803 & $\mathrm{C}$ & $\mathrm{H}^{\mathrm{i}}$ & $\bullet$ & \\
\hline Ulva prolifera Müller, 1778 & $\mathrm{C}$ & $\mathrm{H}^{\mathrm{is}} / \mathrm{EP}$ & & \\
\hline Ulva ralfsii (Harvey) Le Jolis, 1863 & $\mathrm{C}$ & $\mathrm{H}^{\mathrm{i}} / \mathrm{EP}$ & $\bullet$ & \\
\hline
\end{tabular}

MAGNOLIOPHYTA: LILIOPSIDA (Seagrasses)

Family: Hydrocharitaceae

Enhalus acoroides (Linnaeus) Royle, 1839

Halophila decipiens Ostenfeld, 1902

Halophila minor (Zollinger) den Hartog, 1957

Halophila ovalis (Brown) Hooker, 1859

Halophila spinulosa (Brown) Ascherson, 1875

Thalassia hemprichii (Ehrenberg) Ascherson, 1871

IWP

Cymodocea angustata Ostenfeld, 1916

Cymodocea serrulata (Brown) Ascherson \& Magnus, 1870

IWP

Halodule pinifolia (Miki) den Hartog, 1964

IWP

Halodule uninervis (Forsskål) Ascherson, 1882

Syringodium isoetifolium (Ascherson) Dandy, 1939

Thalassodendron ciliatum (Forsskål) den Hartog, 1970

IWP

IWP

IWP

$\begin{array}{lll}S^{s} & \bullet & \bullet \\ S^{s} & \bullet & \bullet \\ S^{s} & \bullet & \\ S^{s} & \bullet & \bullet \\ S^{s} & \bullet & \\ S^{i s} & \bullet & \end{array}$

MAGNOLIOPHYTA: MAGNOLIOPSIDA (Mangroves)

Family: Euphorbiaceae

Excoecaria agallocha Linnaeus, 1759

IWP

NA 


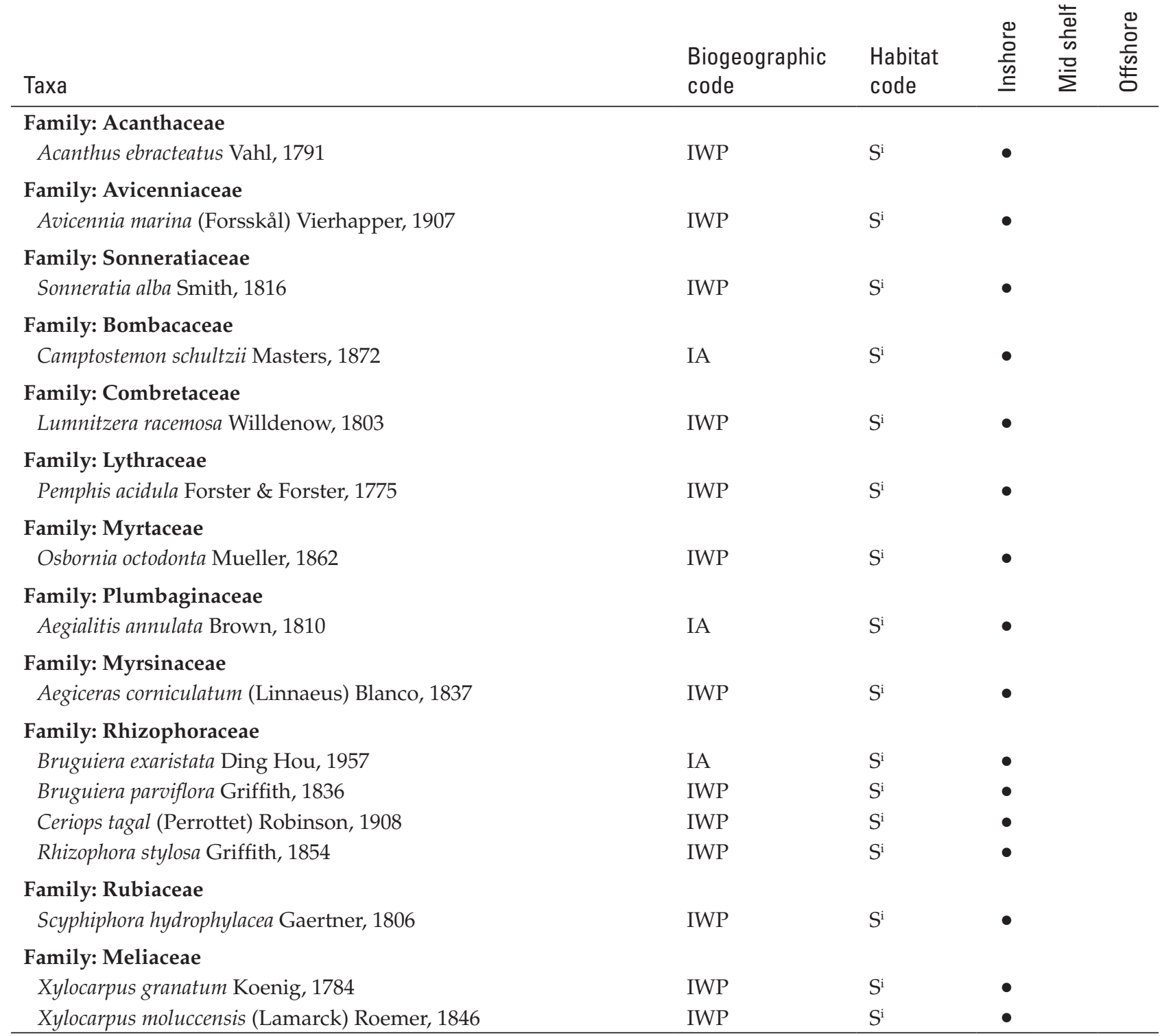


Marine flora data are available for 116 locations in the Kimberley Project Area (Table 4, Figures 1-3). Species richness ranged from 127 (at One Arm Point) to one (at 17 coastal locations) (Table 4, Figure 2). Collecting effort was highly variable with 33 collecting events occurring at One Arm Point and one event at 77 locations (Table 4, Figure 3).

The number of flora taxa collected at any location was also highly variable. All floral taxa (bluegreen, red, brown and green algae, seagrasses and mangroves) were collected at only two locations, One Arm Point and Sunday Island (Table 4). A single floral taxon was collected at 56 locations, red and brown algae at two locations (e.g. Salural Island and Hedley Island respectively), green algae and seagrasses at seven locations (e.g. Browse Island and Allora Island respectively), and mangroves only at 38 locations (e.g. Boongaree Island, Table 4).

More species have been reported from the Kimberley coast (inshore, 241 species) compared to the offshore atolls (124 species, Tables 3, 6, 7). Only two species have been recorded from the midshelf (Browse Island). However, collecting effort is also substantially different for these areas of the shelf, with more collecting of marine plants along the Kimberley coastline compared to the offshore atolls or midshelf (81 collecting events versus 7 and 2 respectively, Table 4). Composition of the flora also varied across the shelf, with 185 species found only at inshore locations, 68 at offshore locations and 56 species at both inshore and offshore locations (Table 3).

\section{BIOGEOGRAPHY}

The majority of the species at both inshore and offshore locations were widespread Indo-West Pacific (IWP) species (102, 42\% inshore, versus 41, $33 \%$ offshore, Table 5 ).

Endemicity was much higher along the Kimberley coast than at the offshore atolls. The proportion of endemics (all categories WA, A, and NA) along the Kimberley coast was twice the proportion of endemics at the offshore atolls (11, 5\% inshore versus $3,2.5 \%$ offshore, Table 5 ).

The majority of seagrass species were IWP (10 species), one tropicopolitan species and a single endemic, Cymodocea angustata Ostenfeld, 1916. The majority of mangrove species were wide ranging IWP (14 species), 3 species were IA, the only marine flora taxa in this category, and there was a single northern Australian endemic species, Excoecaria ovalis Endl., 1833 (Table 3).

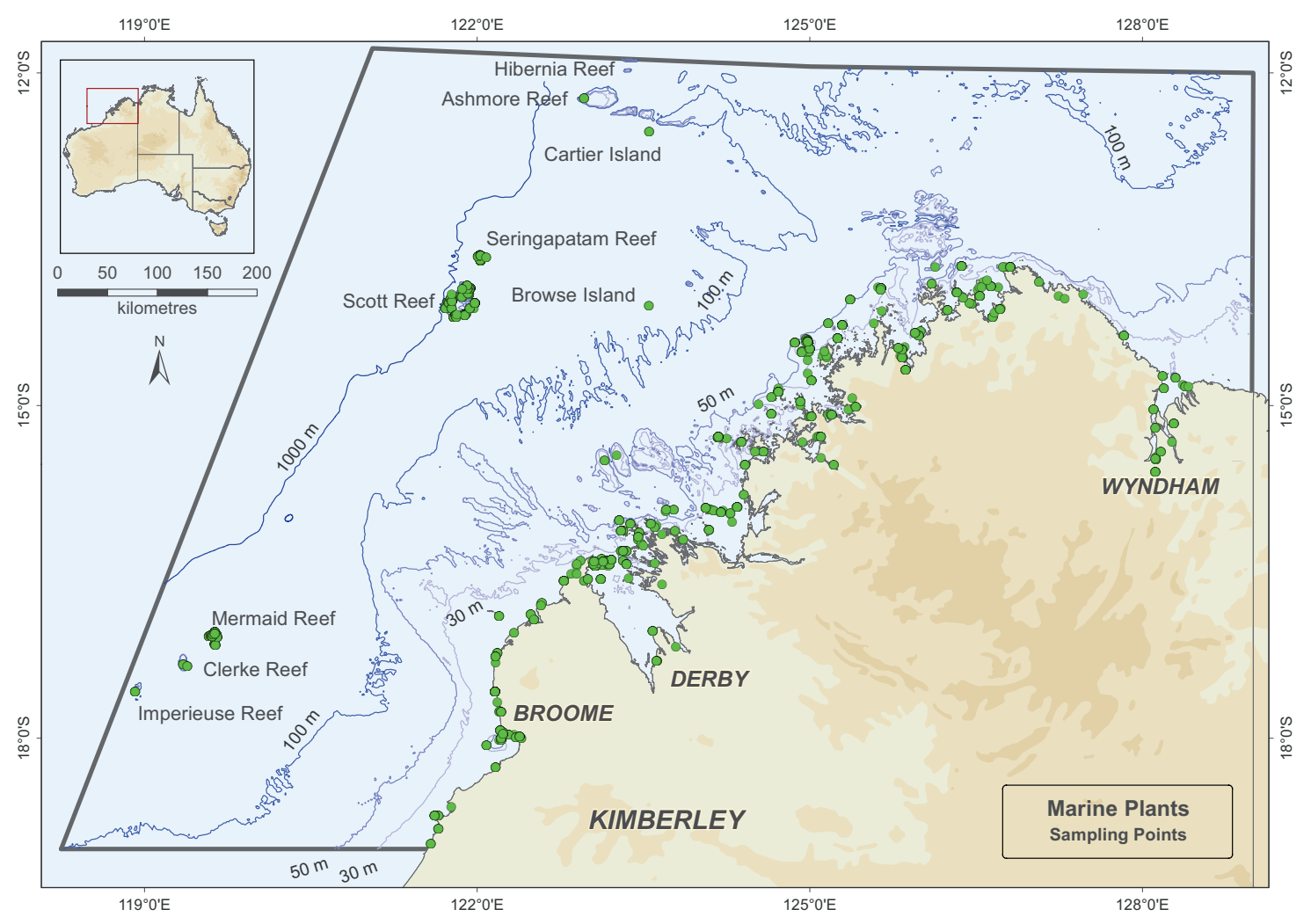

FIGURE 1 Location of records of marine flora in the Kimberley Project Area, Western Australia. The Project Area boundary is marked in grey. Map projection: GDA94, Scale: 1:6, 250,000. 


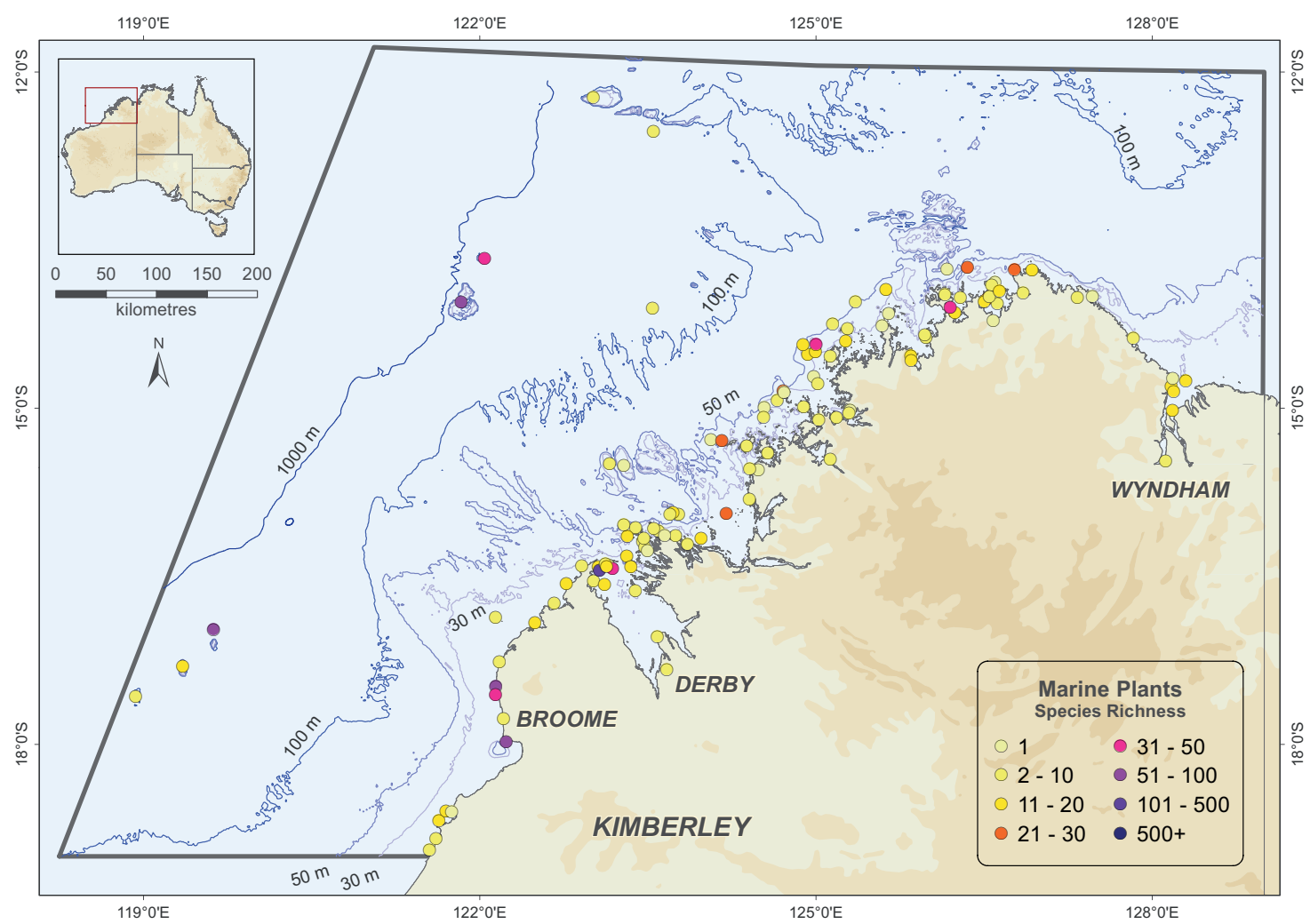

FIGURE 2 Species richness of marine flora for each main location. Map projection: GDA94, Scale: 1:6, $250,000$.

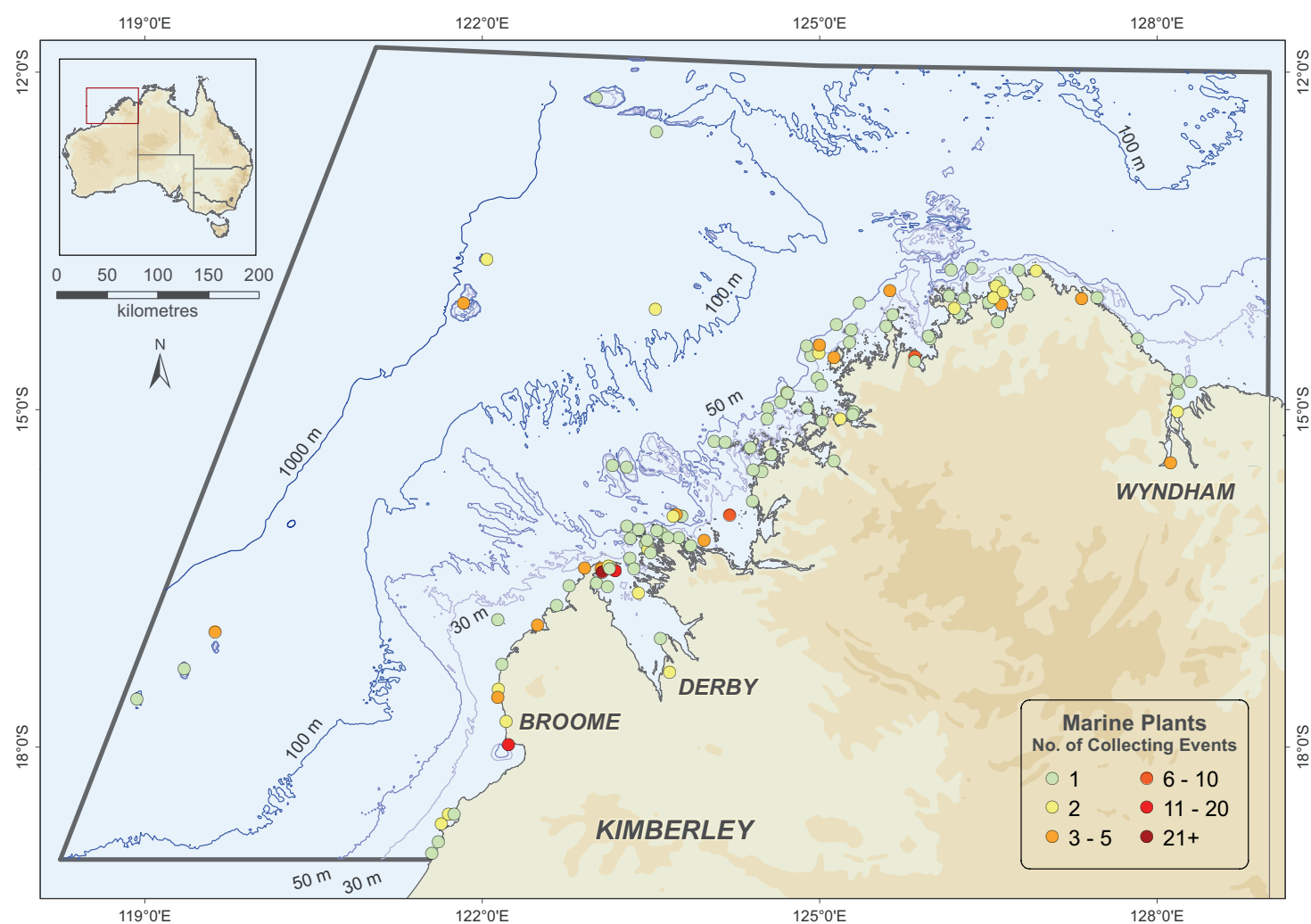

FIGURE 3 Number of collecting events for marine flora at each main location for which there are collections. This was based on a count of the season code and provides an indication of sampling effort. Map projection: GDA94, Scale: 1:6, 250,000. 
TABLE 4 Species richness and number of collecting events at each location.

\begin{tabular}{|c|c|c|c|c|c|c|c|c|c|c|}
\hline Location & $\begin{array}{l}\text { Collecting } \\
\text { year range }\end{array}$ & 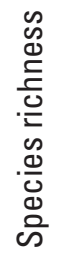 & $\begin{array}{l}\frac{\infty}{c} \\
\frac{0}{\Phi} \\
\searrow \\
\overline{\bar{O}} \\
0 \\
\dot{Z}\end{array}$ & $\begin{array}{l}\underset{x}{x} \\
\stackrel{\pi}{ \pm} \\
\dot{0}\end{array}$ & 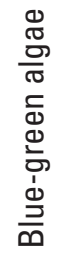 & 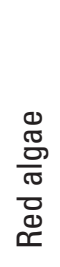 & 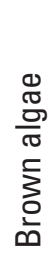 & 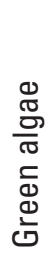 & $\begin{array}{l}\mathscr{d} \\
\mathscr{D} \\
\mathbb{\Xi} \\
\text { ర్ర } \\
\mathbb{\Xi} \\
\mathscr{d}\end{array}$ & 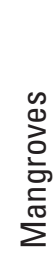 \\
\hline Adele Island & 1990 & 3 & 1 & 1 & & & & $\bullet$ & & \\
\hline Admiral Bay & - & 5 & 1 & 1 & & & & & & $\bullet$ \\
\hline Admiral Island & 1994 & 7 & 1 & 3 & & $\bullet$ & & & $\bullet$ & $\bullet$ \\
\hline Albert Islands & 2007 & 14 & 1 & 3 & & $\bullet$ & $\bullet$ & $\bullet$ & & \\
\hline Allora Island & 1985 & 1 & 1 & 1 & & & & & $\bullet$ & \\
\hline Ashmore Reef ${ }^{\mathrm{OA}}$ & - & 5 & 1 & 1 & & & & & $\bullet$ & \\
\hline Augustus Island & 1972 & 4 & 1 & 1 & & & & & & $\bullet$ \\
\hline Beagle Bay & 1959-1996 & 11 & 4 & 3 & & & & $\bullet$ & $\bullet$ & $\bullet$ \\
\hline Bedford Island & 1994 & 16 & 1 & 5 & & $\bullet$ & $\bullet$ & $\bullet$ & $\bullet$ & $\bullet$ \\
\hline Berthier Island & 1996-2007 & 12 & 2 & 5 & & $\bullet$ & $\bullet$ & $\bullet$ & $\bullet$ & $\bullet$ \\
\hline Bigge Island & 1972-2003 & 4 & 3 & 1 & & & & & & $\bullet$ \\
\hline Boongaree Island & 1973-1988 & 8 & 2 & 1 & & & & & & $\bullet$ \\
\hline Broome & 1889-2007 & 61 & 18 & 5 & & $\bullet$ & $\bullet$ & $\bullet$ & $\bullet$ & $\bullet$ \\
\hline Browse Island ${ }^{\mathrm{MS}}$ & 2006 & 2 & 2 & 1 & & & & $\bullet$ & & \\
\hline Caffarelli Island & 1994 & 10 & 1 & 3 & & $\bullet$ & $\bullet$ & & $\bullet$ & \\
\hline Cambridge Gulf & 1978-1995 & 18 & 2 & 4 & & $\bullet$ & $\bullet$ & $\bullet$ & & $\bullet$ \\
\hline Camden Sound & 1967 & 1 & 1 & 1 & & & & & & $\bullet$ \\
\hline Cape Bernier & 1988 & 1 & 1 & 1 & & & & & & $\bullet$ \\
\hline Cape Bertholet & 1977 & 10 & 1 & 1 & & & & & & $\bullet$ \\
\hline Cape Bossut & 1977-1985 & 13 & 2 & 4 & & $\bullet$ & $\bullet$ & $\bullet$ & & $\bullet$ \\
\hline Cape Bougainville & - & 3 & 1 & 1 & & & & & & $\bullet$ \\
\hline Cape Domett & 1995 & 14 & 1 & 3 & & $\bullet$ & & $\bullet$ & & $\bullet$ \\
\hline Cape Dussejour & 1978 & 1 & 1 & 1 & & & & & & $\bullet$ \\
\hline Cape Jaubert & - & 4 & 1 & 1 & & & & & & $\bullet$ \\
\hline Cape Leveque & 1977-2000 & 6 & 5 & 3 & & & $\bullet$ & & $\bullet$ & $\bullet$ \\
\hline Cape Londonderry & 1975-1995 & 19 & 2 & 5 & & $\bullet$ & $\bullet$ & $\bullet$ & $\bullet$ & $\bullet$ \\
\hline Cape Talbot & 1995 & 24 & 1 & 4 & & $\bullet$ & $\bullet$ & $\bullet$ & & $\bullet$ \\
\hline Cape Voltaire & 1976 & 1 & 1 & 1 & & & & & & $\bullet$ \\
\hline Careening Bay & 1972 & 5 & 1 & 1 & & & & & & $\bullet$ \\
\hline Carlia Island & - & 8 & 1 & 1 & & & & & & $\bullet$ \\
\hline Cartier Island ${ }^{\mathrm{OA}}$ & 1977 & 3 & 1 & 3 & & $\bullet$ & & $\bullet$ & $\bullet$ & \\
\hline Cassini Island & 1991-1996 & 18 & 3 & 4 & & $\bullet$ & $\bullet$ & $\bullet$ & $\bullet$ & \\
\hline Champagny Island & 1972 & 1 & 1 & 1 & & & & & & $\bullet$ \\
\hline Churchill Reef & 1996 & 1 & 1 & 1 & & & & $\bullet$ & & \\
\hline Clerke Reef ${ }^{\mathrm{OA}}$ & 2007 & 11 & 1 & 3 & & $\bullet$ & $\bullet$ & $\bullet$ & & \\
\hline Cockatoo Island & 2004 & 4 & 1 & 1 & & & & $\bullet$ & & \\
\hline Colbert Island & 1993-1996 & 23 & 2 & 4 & & $\bullet$ & $\bullet$ & $\bullet$ & $\bullet$ & \\
\hline Coronation Island & 1972 & 8 & 1 & 1 & & & & & & $\bullet$ \\
\hline
\end{tabular}




\begin{tabular}{|c|c|c|c|c|c|c|c|c|c|c|}
\hline Location & $\begin{array}{l}\text { Collecting } \\
\text { year range }\end{array}$ & 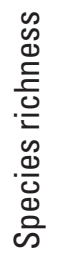 & 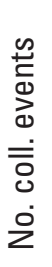 & $\begin{array}{l}\underset{\pi}{x} \\
\underset{t}{+} \\
\dot{0} \\
\dot{Z}\end{array}$ & 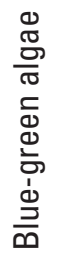 & 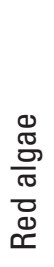 & 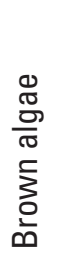 & $\begin{array}{l}0 \\
\mathbb{J} \\
\frac{\sigma}{\pi} \\
\frac{\sigma}{0} \\
\frac{d}{0} \\
\frac{1}{0}\end{array}$ & 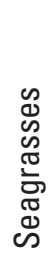 & 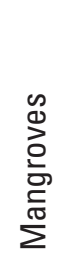 \\
\hline Cygnet Bay & 1977 & 5 & 1 & 1 & & & & & & $\bullet$ \\
\hline Darcy Island & - & 8 & 1 & 1 & & & & & & $\bullet$ \\
\hline De Freycinet Island & 1996 & 1 & 1 & 1 & & $\bullet$ & & & & \\
\hline Derby & 1971-1980 & 8 & 2 & 1 & & & & & & $\bullet$ \\
\hline Drysdale River & 1995 & 10 & 1 & 1 & & & & & & $\bullet$ \\
\hline False Cape Bossut & 2001 & 19 & 2 & 3 & & $\bullet$ & $\bullet$ & $\bullet$ & & \\
\hline Freshwater Bay & 1995 & 8 & 1 & 1 & & & & & & $\bullet$ \\
\hline Freshwater Cove & 2001 & 3 & 1 & 1 & & & & $\bullet$ & & \\
\hline Gagg Island & 1994 & 5 & 1 & 1 & & & & & $\bullet$ & \\
\hline Gibbings Reefs & 1996 & 3 & 1 & 2 & & $\bullet$ & & $\bullet$ & & \\
\hline Gregory Island & 1994 & 12 & 1 & 5 & & $\bullet$ & $\bullet$ & $\bullet$ & $\bullet$ & $\bullet$ \\
\hline Hedley Island & 1996 & 2 & 1 & 1 & & & $\bullet$ & & & \\
\hline Heritage Reef & 1996 & 6 & 1 & 3 & & & $\bullet$ & $\bullet$ & $\bullet$ & \\
\hline Hidden Island & 1982-1994 & 5 & 2 & 3 & & $\bullet$ & $\bullet$ & & & $\bullet$ \\
\hline Imperieuse Reef $^{\mathrm{OA}}$ & 2007 & 6 & 1 & 4 & & $\bullet$ & $\bullet$ & $\bullet$ & $\bullet$ & \\
\hline Irvine Island & 1994 & 6 & 1 & 3 & & $\bullet$ & $\bullet$ & & $\bullet$ & \\
\hline Jackson Island & 2003-2005 & 13 & 5 & 2 & & $\bullet$ & & $\bullet$ & & \\
\hline James Price Point & 1988-2009 & 76 & 2 & 5 & $\bullet$ & $\bullet$ & $\bullet$ & $\bullet$ & $\bullet$ & \\
\hline Jamieson Reef & 1996 & 5 & 1 & 3 & & $\bullet$ & $\bullet$ & $\bullet$ & & \\
\hline Jar Island & 1995 & 14 & 1 & 3 & & $\bullet$ & $\bullet$ & $\bullet$ & & \\
\hline Jones Island & 1995 & 21 & 1 & 3 & & $\bullet$ & $\bullet$ & $\bullet$ & & \\
\hline Jussieu Island & 1996 & 1 & 1 & 1 & & & $\bullet$ & & & \\
\hline King Edward River & 1995 & 1 & 1 & 1 & & & & $\bullet$ & & \\
\hline King George River & 1992-1995 & 9 & 2 & 1 & & & & & & $\bullet$ \\
\hline King Sound & 1977-1996 & 2 & 2 & 1 & & & & & & $\bullet$ \\
\hline Kingfisher Island & 1990-2001 & 13 & 3 & 3 & & $\bullet$ & $\bullet$ & & & $\bullet$ \\
\hline Koolan Island & 1993 & 3 & 1 & 1 & & & & & & $\bullet$ \\
\hline Lacepede Islands & 1986 & 6 & 1 & 2 & & & $\bullet$ & $\bullet$ & & \\
\hline Lacrosse Island & 1995 & 11 & 1 & 3 & & $\bullet$ & & $\bullet$ & & $\bullet$ \\
\hline Lagrange Bay & - & 1 & 1 & 1 & & & & & & $\bullet$ \\
\hline Lamarck Island & 1996 & 5 & 1 & 3 & & $\bullet$ & $\bullet$ & $\bullet$ & & \\
\hline Leonie Island & 1985-2002 & 11 & 3 & 4 & & $\bullet$ & $\bullet$ & $\bullet$ & $\bullet$ & \\
\hline Long Island & 1982-1995 & 31 & 2 & 5 & & $\bullet$ & $\bullet$ & $\bullet$ & $\bullet$ & $\bullet$ \\
\hline Lord Island & 1994 & 3 & 1 & 2 & & & $\bullet$ & & $\bullet$ & \\
\hline Louis Islands & 1995 & 27 & 1 & 5 & & $\bullet$ & $\bullet$ & $\bullet$ & $\bullet$ & $\bullet$ \\
\hline Mackenzie Anchorage & 1995 & 13 & 1 & 2 & & & & $\bullet$ & & $\bullet$ \\
\hline Macleay Island & 1994-1996 & 6 & 2 & 4 & $\bullet$ & $\bullet$ & $\bullet$ & & & $\bullet$ \\
\hline Maret Islands & 1996-2007 & 38 & 4 & 4 & & $\bullet$ & $\bullet$ & $\bullet$ & $\bullet$ & \\
\hline Mermaid Island & 1994 & 16 & 1 & 5 & & $\bullet$ & $\bullet$ & $\bullet$ & $\bullet$ & $\bullet$ \\
\hline
\end{tabular}




\begin{tabular}{|c|c|c|c|c|c|c|c|c|c|c|}
\hline Location & $\begin{array}{l}\text { Collecting } \\
\text { year range }\end{array}$ & 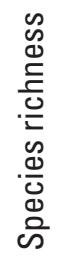 & 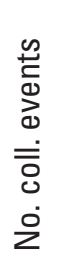 & 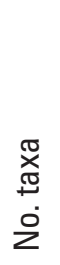 & 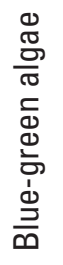 & $\begin{array}{l}0 \\
\frac{\pi}{\pi} \\
\frac{0}{\pi} \\
\frac{0}{0} \\
\approx\end{array}$ & 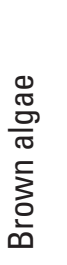 & $\begin{array}{l}\mathbb{\Xi} \\
\frac{\pi}{\pi} \\
\frac{\pi}{\pi} \\
\frac{1}{\Phi} \\
\frac{0}{\omega}\end{array}$ & 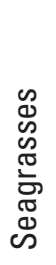 & 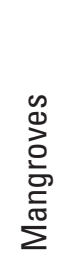 \\
\hline Mermaid Reef ${ }^{\mathrm{OA}}$ & $2006-2007$ & 64 & 3 & 4 & & $\bullet$ & $\bullet$ & - & $\bullet$ & \\
\hline Montalivet Islands & 1996 & 10 & 1 & 3 & & $\bullet$ & $\bullet$ & - & & \\
\hline Montgomery Reef & 1987-2005 & 28 & 6 & 5 & & $\bullet$ & $\bullet$ & $\bullet$ & $\bullet$ & $\bullet$ \\
\hline Myrmidon Ledge & 1995 & 17 & 1 & 4 & & $\bullet$ & $\bullet$ & $\bullet$ & & $\bullet$ \\
\hline Napier Broome Bay & 1921-1999 & 7 & 5 & 1 & & & & & & $\bullet$ \\
\hline Naturalists Island & 1997 & 1 & 1 & 1 & & & & $\bullet$ & & \\
\hline One Arm Point & 1984-2006 & 127 & 33 & 6 & $\bullet$ & $\bullet$ & - & $\bullet$ & $\bullet$ & $\bullet$ \\
\hline Osborne Islands & 1973 & 8 & 1 & 1 & & & & & & $\bullet$ \\
\hline Packer Island & 1977 & 11 & 1 & 1 & & & & & & $\bullet$ \\
\hline Pender Bay & 2008 & 3 & 1 & 1 & & & & & & $\bullet$ \\
\hline Point Torment & - & 9 & 1 & 1 & & & & & & $\bullet$ \\
\hline Port Warrender & 1976-2004 & 13 & 7 & 1 & & & & & & $\bullet$ \\
\hline Prince Frederick Harbour & 1984 & 7 & 1 & 1 & & & & & & $\bullet$ \\
\hline Prince Regent River & 1974 & 10 & 1 & 1 & & & & & & $\bullet$ \\
\hline Prudhoe Islands & 1996 & 12 & 1 & 3 & & $\bullet$ & - & $\bullet$ & & \\
\hline Quondong Point & 2001 & 45 & 3 & 4 & & $\bullet$ & - & $\bullet$ & $\bullet$ & \\
\hline Reveley Island & 1995 & 10 & 1 & 3 & & $\bullet$ & - & $\bullet$ & & \\
\hline Robroy Reefs & 1996 & 11 & 1 & 3 & & $\bullet$ & $\bullet$ & $\bullet$ & & \\
\hline Salural Island & 2003-2004 & 3 & 2 & 1 & & $\bullet$ & & & & \\
\hline Scorpion Island & 1991 & 1 & 1 & 1 & & & & & $\bullet$ & \\
\hline Scott Reef ${ }^{\mathrm{OA}}$ & 2006-2007 & 88 & 4 & 5 & $\bullet$ & $\bullet$ & - & $\bullet$ & $\bullet$ & \\
\hline Seringapatam Reef ${ }^{\mathrm{OA}}$ & 2006 & 46 & 2 & 5 & $\bullet$ & $\bullet$ & $\bullet$ & $\bullet$ & • & \\
\hline Sir Graham Moore Island & 1973-1975 & 3 & 2 & 2 & & $\bullet$ & & & & $\bullet$ \\
\hline Slate Islands & 1996 & 7 & 1 & 4 & & $\bullet$ & $\bullet$ & $\bullet$ & $\bullet$ & \\
\hline Sunday Island & 1982-2005 & 37 & 11 & 6 & $\bullet$ & $\bullet$ & - & $\bullet$ & $\bullet$ & $\bullet$ \\
\hline Talbot Bay & 1994 & 3 & 1 & 2 & & & $\bullet$ & & $\bullet$ & \\
\hline Tallon Island & 1994 & 11 & 1 & 4 & & $\bullet$ & - & & $\bullet$ & $\bullet$ \\
\hline Trent River & - & 11 & 1 & 1 & & & & & & $\bullet$ \\
\hline Troughton Island & 1999 & 1 & 1 & 1 & & & & & & $\bullet$ \\
\hline Vansittart Bay & 1921-1984 & 2 & 2 & 1 & & & & & & $\bullet$ \\
\hline Walsh Point & - & 11 & 1 & 1 & & & & & & $\bullet$ \\
\hline West Governor Island & 1984-1995 & 17 & 2 & 4 & & $\bullet$ & $\bullet$ & - & & $\bullet$ \\
\hline Whirlpool Pass & 1994 & 1 & 1 & 1 & & & & & $\bullet$ & \\
\hline White Island & 1996 & 6 & 1 & 2 & & $\bullet$ & $\bullet$ & & $\bullet$ & \\
\hline Wildcat Reefs & 1996 & 25 & 1 & 4 & & $\bullet$ & $\bullet$ & $\bullet$ & & \\
\hline Willie Creek & 1978-2007 & 8 & 2 & 1 & & & & & & $\bullet$ \\
\hline Woodward Island & 1991 & 1 & 1 & 1 & & & & & $\bullet$ & \\
\hline Wyndham & 1974-1978 & 4 & 3 & 1 & & & & & & $\bullet$ \\
\hline Yankawingarri Island & 1991 & 1 & 1 & 1 & & & & & $\bullet$ & \\
\hline
\end{tabular}


TABLE $5 \quad$ Number of species with each biogeographic code.

\begin{tabular}{|c|c|c|c|}
\hline Biogeographic Code & Inshore & Midshelf & Offshore \\
\hline- & 10 & & 2 \\
\hline WA & 6 & & 2 \\
\hline NA & 3 & & 1 \\
\hline A & 2 & & \\
\hline IA & 3 & & \\
\hline $\mathrm{IO}$ & 2 & & 2 \\
\hline IWP & 102 & 1 & 41 \\
\hline IP & 9 & & 4 \\
\hline $\mathrm{T}$ & 65 & 1 & 40 \\
\hline C & 39 & & 32 \\
\hline Total & 202 & 2 & 92 \\
\hline
\end{tabular}

TABLE 6 Number of species with each habitat code.

\begin{tabular}{|c|c|c|c|}
\hline Habitat Code & Inshore & Midshelf & Offshore \\
\hline $\mathrm{EnP}$ & & & 2 \\
\hline $\mathrm{EP}$ & 10 & & 17 \\
\hline $\mathrm{H} / \mathrm{S}^{\mathrm{i}}$ & 3 & & \\
\hline $\mathrm{H} / \mathrm{S}^{\mathrm{s}}$ & 1 & & \\
\hline $\mathrm{H}^{\mathrm{i}}$ & 48 & 2 & 15 \\
\hline $\mathrm{H}^{\mathrm{i}} / \mathrm{EP}$ & 6 & & 5 \\
\hline $\mathrm{H}^{\mathrm{is}}$ & 60 & & 31 \\
\hline $\mathrm{H}^{\text {is }} / \mathrm{EP}$ & 11 & & 7 \\
\hline $\mathrm{H}^{\mathrm{is}} / \mathrm{S}^{\mathrm{i}}$ & 1 & & \\
\hline $\mathrm{H}^{\mathrm{s}}$ & 51 & & 32 \\
\hline $\mathrm{H}^{\mathrm{s}} / \mathrm{EP}$ & & & 1 \\
\hline $\mathrm{H}^{\mathrm{s}} / \mathrm{S}^{\text {is }}$ & 1 & & 1 \\
\hline $\mathrm{S}^{\mathrm{i}}$ & 22 & & 2 \\
\hline $\mathrm{S}^{\text {is }}$ & 10 & & 7 \\
\hline$S^{s}$ & 13 & & 4 \\
\hline $\mathrm{U}$ & 4 & & \\
\hline Total & 241 & 2 & 124 \\
\hline
\end{tabular}

HABITATS

The majority of the species surveyed in the Project Area were associated with hard substrates, 214 inshore versus 101 offshore for all combinations $\left(\mathrm{H} / \mathrm{S}^{\mathrm{i}}, \mathrm{H} / \mathrm{S}^{\mathrm{s}}, \mathrm{H}^{\mathrm{i}}, \mathrm{H}^{\mathrm{i}} / \mathrm{EP}, \mathrm{H}^{\mathrm{is}}, \mathrm{H}^{\mathrm{is}} / \mathrm{EP}\right.$, and $\left.\mathrm{H}^{\mathrm{s}} / \mathrm{S}^{\mathrm{is}}\right)$. A small proportion of species that occurred on hard substrates were also found on soft substrates (6 species, Tables 3, 6).

The number of species that only occur on soft substrates was much lower, 51 species from inshore and 14 from offshore locations (Table 6). All mangrove and seagrass species were associated with soft substrates. All mangroves species (18) were in soft sediment intertidal areas.

Only two algae species were endophytic in other algae (EnP), the red alga Pihiella liagoraciphila Huisman, Sherwood and Abbott, 2003 occurred in various species of Liagoraceae, and the green Blastophysa rhizopus Reinke, 1889 in some softbodied red algae (Tables 3, 6). Forty eight species of algae were epiphytic, some also associated with hard substrate.

\section{DISCUSSION}

The present compendium and analysis provides a general overview of the Kimberley Project Area marine flora, but there are several caveats regarding the collection methods and post collection handling of specimens used to generate the results, notably:

1. Collecting effort is variable, depending on the location, the collector, and the conditions at the time of collection. Recent expeditions with WAM have standardised the methods, but many of the earlier collections were more haphazard.

2. The taxonomy of many groups is unresolved. This is due to several factors both practical and scientific, including a lack of available expertise, time constraints, and the now universal use of DNA sequencing, which is altering many taxonomic concepts. Recent surveys have incorporated the appropriate preservation of specimens for DNA extraction, but in the past all specimens were ritually preserved in formalin, thus making them unsuitable for such analyses. A typical example of the changes occurring is the filamentous red alga previously known as Ceramium flaccidum and thought to be morphologically variable and cosmopolitan in distribution. The study by Cho et al. (2008) incorporated DNA analyses to support their morphological observations and recognised seven species within the $C$. flaccidum complex, which they transferred to the new genus Gayliella. Ceramium flaccidum sensu lato was recorded for the Project Area offshore atolls by Huisman et al. (2009) and 
generally throughout north-western Australia. Based on the conclusions of Cho et al. (2008) a re-examination of the specimens has revealed five species, including one new and two yet to be transferred from Ceramium. True Ceramium flaccidum (= Gayliella flaccida) does not occur in north-western Australia. Similar DNA sequence based revisions are occurring in a large number of taxa.

3. Much of the species diversity, particularly in the red algae, is due to the essentially microscopic epiphytes. These generally cannot be recognised while collecting, and only become apparent when larger species are examined in the laboratory. As a result, records of these species can be erratic and possibly not comparable between locations.

4. Many specimens cannot be named for a variety of reasons. Identification of many of the red algae requires reproductive material, and in most cases this cannot be assessed while collecting. It can, therefore, be a matter of luck if suitable material is collected. DNA sequencing assists this process considerably, but can only provide identification if a matching sequence of a reliably named specimen is available for comparison.

5. The marine flora of the Kimberley Project Area has not yet received the attention enjoyed by some faunal groups and has only relatively recently been collected and studied in any detail.

Given the above overarching limitations, it is almost impossible to recognise diversity patterns that are not compromised in some way, and the following broad discussion is perhaps most useful in highlighting collection gaps, both geographical and habitat.

\section{SPECIES RICHNESS PATTERNS}

A summary of macroalgal species richness for various regions in the Indo-West Pacific was given by Huisman et al. (2009: Table 3). The present dataset records 278 species, an increase on the number of Kimberley species recorded in that publication, but still undoubtedly an underestimate given the large number of excluded lots. Currently the first author is compiling an algal flora for tropical north-western Australia, anticipated to be published by the 'Australian Biological Resources Study' in 2015 (Huisman, in preparation). This publication will document the northern Gascoyne, Pilbara and Kimberley marine algal floras including full descriptions and illustrations of all red, green and brown algae from the region. The manuscript includes some 413 species (262 red algae, 55 brown algae, 96 green algae) of which approximately 50 are new species. These new species are not represented in the present dataset. Verheij and Prud'homme van Reine (1993) recorded 452 species for Indonesia, and given the proximity and comparable range of habitats, the Project Area algal flora is likely to include a similar number.

Over the last two decades, the relatively static numbers of seagrasses and mangroves recorded for the region suggests that the known diversity is an accurate assessment of the flora. These groups do not include any inconspicuous taxa and are relatively taxonomically stable (at least for the Project Area species), so regional scale changes to the recorded diversity are unlikely. Compared to the macroalgae, for seagrasses (12 species) and mangroves (18 species) the diversity is low, but these groups are generally conspicuous and can dominate particular habitats. Relative to other Indo-West Pacific regions, the north-west seagrass diversity is the highest recorded (Walker and Prince 1987: Table 5).

Worldwide, some 72 species of mangrove are known and more than half of these can be found in Australia, where almost 18 per cent of the coastline is lined by mangroves. Western Australia supports approximately 2,500 square kilometres of mangroves, of which about 75 per cent occur in the Kimberley (Duke 2006). The present dataset includes 18 species for the region, one less than the number recorded by Duke (2006). This diversity is less than that known for north eastern Australia, but the region's mangroves occur in a diverse array of habitats, and are nevertheless of great conservation significance (Cresswell and Semeniuk 2011).

The Project Area includes seven IMCRA mesoscale bioregions (Commonwealth of Australia 2006) and therefore encompasses a variety of habitats, from sandy shores to offshore reefs. These regions were defined using biological and physical information, including the distribution of demersal fishes, marine plants and invertebrates, seafloor geomorphology and sediments, and oceanographic data (Commonwealth of Australia 2006). At this scale, the largest disjunct in the Kimberley Project Area marine flora is between the inshore islands and reefs and offshore atolls. The diversity of the offshore atolls is markedly lower, with some groups (such as mangroves) and genera (such as the brown alga Sargassum) absent entirely. Lack of suitable habitat can explain the absence of mangroves, but it is unclear why Sargassum, easily the dominant alga in the inshore Kimberley, is absent from the atolls. Studies by Schaffelke (1999) and Schaffelke and Klumpp (1998) on eastern Australian Sargassum species have demonstrated their increased growth with nutrient pulses and their use of particulate 
matter as a nutrient source. Perhaps the offshore atolls are too far removed from land derived nutrients. Clearly further study is needed.

Species diversity can also be expected to vary within regions, as it is highly dependent on finer scale habitat variations. For macroalgae there are numerous examples of sites dominated by algae and with a high species diversity (usually intertidal/shallow subtidal reef flats), and also of others almost devoid of algae (usually moderate to deep subtidal, often with high turbidity). The habitat factors driving these fine scale variations cannot be assessed from the present historical dataset, but current and future WAM expeditions will assess a greater variety of physical and biological parameters.

\section{COLLECTION GAPS}

The Kimberley is a vast, remote wilderness. The limitations on marine collecting are numerous, including physical (tides, accessibility, turbid water, harsh climate), biological (sharks, crocodiles), financial (travel, boat charter), and health and safety (limited dive times). As such, it is inevitable that some areas have not been surveyed fully, if at all. Some, such as Ashmore Reef and Cartier Island, were visited by WAM prior to the participation of a marine botanist, and therefore represent significant spatial gaps in our knowledge of the marine flora. Certain habitats are equally under represented. Some, such as the deep seafloor, require specialised dredging equipment that can generally only be accommodated on dedicated surveys. Others, such as the subtidal inshore, are unlikely to be sampled due to safety concerns.

Of these gaps, those that can feasibly be sampled will be targeted during current WAM expeditions.

\section{ENDEMISM}

The present dataset records endemism at between 2.5 and $5 \%$, but this is an underestimate as it does not include undescribed new taxa. Huisman (2011) estimated endemism in the north-western Australian macroalgal flora at approximately $10 \%$, which encompasses all the taxa to be described in Huisman (in preparation) plus a few previously erected species and genera. This percentage is low relative to those recorded for the temperate southern Australian algal flora, which Womersley (1984) gave as 30\% (green algae), 70\% (brown algae) and $75 \%$ (red algae). This disparity is to be expected as many tropical species are broadly distributed, particularly in the Indo-West Pacific, and temperate regions typically have a greater proportion of endemic species. However, our documentation of this flora is still a work in progress and many specimens remain unidentified, this group including the more 'difficult' taxa. As such, an accurate assessment of endemism can only be made once all the specimens are identified. Whether these taxa are truly endemic will require considerable work, including collections from further north in SE Asia. Assessing endemism in the marine flora is a difficult process and we should heed the cautionary words of Kraft (2007), who stated, 'Determining actual endemism among marine algae, particularly subtidal ones, is a greater challenge than it is for most terrestrial taxa, and claims made for the uniqueness of a region based on its number of endemic macroalgal species can easily prove not to be justified'.

\section{FUTURE DIRECTIONS}

The present dataset has highlighted several areas that are underrepresented in the Western Australian Herbarium's collection of marine algae, in particular. Filling these gaps during current WAM expeditions is therefore a high priority. Of comparable importance is the identification of the 1,342 (or close to 50\%) of the Project Area specimens, which were excluded from the present assessment due to their incomplete identifications. These specimens potentially contain useful information about the marine flora of the Kimberley Project Area. Identification of these specimens is an ongoing task of the first author, but is a huge undertaking, which might consume his whole working life, and then some. Taxonomists regularly highlight dwindling resources and the lack of support for their work, and this malaise is true for marine floristic studies. Continued (or some!) funding support for these studies is essential.

Equally important is the incorporation of DNA sequencing as a tool enabling a greater degree of confidence in taxonomic studies. During recent WAM expeditions, specimens were curated specifically for DNA sequencing and it is envisaged that this aspect will continue to be a high priority during future expeditions.

Assessing distribution patterns amongst the marine flora, and the marine biota in general, will be greatly enhanced by the collection of targeted environmental data. Particularly relevant to the marine biota would be the assessment of the nutrient status at each site, plus detailed data on habitat and geomorphology. These data could feed into large scale biogeographic analyses for example, looking at the species occurring in the various IMCRA bioregions. At present, and highlighted by the gaps in the present dataset, this cannot be undertaken for the marine flora with any degree of confidence. 


\section{ACKNOWLEDGEMENTS}

Thanks to our taxonomic colleagues, D. Walker, B.J. Carter, R. Johnstone, S. Montgomery, K. McMahon and K. Kenneally for their significant contribution to the collections of specimens contained in this dataset. Thanks to Stacey Osborne and Albert Miles (WAM) for checking taxonomic and spatial information of the records contained in this dataset, and to Stacey Osborne for assistance in formatting tables for this paper. Thanks to Alex Chapman (PERTH) for providing data from the PERTH database.

\section{REFERENCES}

ABRS. (2011). 'Flora of Australia Online.' Retrieved 2010-2011, from http://www.environment.gov.au/ biodiversity /abrs/online-resources / flora/main / index.html.

Brown, K. and Skewes, T. (2005). A preliminary assessment of the ecology of seagrasses at Ashmore Reef. The Beagle, Records of the Museum and Art Galleries of the Northern Territory Supplement 1: 143-152.

Cho, T., Boo, S., Hommersand, M., Maggs, C., McIvor, L. and Fredericq, S. (2008). Gayliella gen. nov. in the tribe Ceramieae (Ceramiaceae, Rhodophyta) based on molecular and morphological evidence. Journal of Phycology 44: 721-738.

Commonwealth of Australia (2006). A Guide to the integrated marine and coastal regionalisation of Australia version 4.0. Canberra, Australia, Department of the Environment and Heritage.

Cowan, R. (2011). 'Australian marine algal name index.' Retrieved 2010-2011 from http://www.anbg.gov.au/ amanisearch/servlet/amanisearch/.

Cresswell, I. and Semeniuk, V. (2011). Mangroves of the Kimberley coast: ecological patterns in a tropical ria coast setting. Journal of the Royal Society of Western Australia 94: 213-237.

Department of Environment and Conservation (2009). Protecting the Kimberley. A synthesis of scientific knowledge to support conservation management in the Kimberley region of Western Australia. Department of Environment and Conservation: Perth.

Duke, N. (2006). Australia's mangroves. The authoritative guide to Australia's mangrove plants. University of Queensland: Brisbane.

Gaston, K.J. and Mound, L.A. (1993). Taxonomy, hypothesis testing and the biodiversity crisis. Proceedings: Biological Sciences 251: 139-142.

Guiry, M. and Guiry, G. (2011). 'AlgaeBase.' Retrieved 2011, from http://www.algaebase.org.

Hanley, J. (1995). Part 6. Mangrove Flora: Distribution of species and habitat descriptions (pp. 67-81). In: Wells, F.E., Hanley, J.R. and Walker, D.I. (eds), Survey of the marine biota of the southern Kimberley islands, Western Australia. Western Australian Museum: Perth. Unpublished Report.

Huisman, J. (2002). The type and Australian species of the red algal genera Liagora and Ganonema (Liagoraceae, Nemaliales). Australian Systematic Botany 15: 773-838.
Huisman, J. (2006). Algae of Australia: Nemaliales. CSIRO Publishing: Melbourne.

Huisman, J. (2011). The Last Frontier: The marine benthic flora of north-western Australia. International Botanical Congress. Melbourne, Victoria.

Huisman, J., Jones, D., Wells, F. and Burton, T. (2008). Introduced marine biota in Western Australian waters. Records Western Australian Museum 25: 1-44.

Huisman, J., Leliaert, F., Verbruggen, H. and Townsend, R. (2009). Marine benthic plants of Western Australia's shelfedge atolls. Records of the Western Australian Museum Supplement 77: 50-88.

Johnstone, R. (1990). Mangrove and Mangrove Birds of Western Australia. Records of the Western Australian Museum Supplement 32: 1-120.

Keesing, J., Irvine, T., Alderslade, P., Clapin, G., Fromont, J., Hosie, A., Huisman, J., Naughton, K., Marsh, L., Slack-Smith, S., Thomson, D. and Watson, J. (2011). Marine benthic flora and fauna of Gourdon Bay and the Dampier Peninsula in the Kimberley region of north-western Australia. Journal of the Royal Society of Western Australia 94: 285-301.

King, R. and Puttock, C. (1994). Macroalgae associated with mangroves in Australia: Rhodophyta. Botancia Marina 37: 181-191.

Kraft, G. (2007). Algae of Australia: Marine benthic algae of Lord Howe Island and the southern Great Barrier Reef, 1: Green algae. CSIRO Publishing: Melbourne.

Leliaert, F., Huisman, J. and Coppejans, E. (2007). Phylogenetic position of Boodlea vanbosseae (Siphonocladales, Chlorophyta). Cryptogamie, Algologie 28: 337-351.

Leliaert, F., Wysor, B., Verbruggen, H., Vlaeminck, C. and De Clerck, O. (2008). Phyllodictyon robustum (Setchell et Gardner) comb. nov. (Siphonocladales, Chlorophyta), a morphologically variable species from the tropical Pacific coast of America. Cryptogamie, Algologie 29: 217-233.

Lewis, J. (1984). Checklist and bibliography of benthic marine macroalgae recorded from northern Australia. I. Rhodophyta. Department of Defense Materials Research Laboratories: Melbourne.

Lewis, J. (1985). Checklist and bibliography of benthic marine macroalgae recorded from northern Australia. II. Phaeophyta. Department of Defense Materials Research Laboratories: Melbourne.

Lewis, J. (1987). Checklist and bibliography of benthic marine macroalgae recorded from northern Australia. III. Chlorophyta. Department of Defense Materials Research Laboratories: Melbourne.

Phillips, J., King, R., Tanaka, J. and Mostaert, A. (1993). Stoechospermum (Dictyotales, Phaeophyceae): a poorly known algal genus newly recorded in Australia. Phycologia 5: 395-398.

Pike, G. and Leach, G. (1997). Handbook of the vascular plants of Ashmore and Cartier Islands. Parks Australia: Canberra.

Pyke, G.H. and Ehrlich, P.R. (2010). Biological collections and ecological/environmental research: a review, some observations and a look to the future. Biological Reviews 85: 247-266.

Saenger, P. (1996). Part 6. Mangrove Flora: Distribution of species and habitat descriptions (pp. 39-53). In: 
Walker, D.I., Wells, F.E. and Hanley, J.R. (eds), Survey of the marine biota of the eastern Kimberley, Western Australia. Western Australian Museum: Perth. Unpublished Report.

Sampey, A., Bryce, C., Osborne, S., Miles, A. (2014). Kimberley marine biota. Historical data: introduction and methods. Records of the Western Australian Museum Supplement 84: 19-43.

Schaffelke, B. (1999). Particulate organic matter as an alternative nutrient source for tropical Sargassum species (Fucales, Phaeophyceae). Journal of Phycology 35: 1150-1157.

Schaffelke, B. and Klumpp, D. (1998). Short-term nutrient pulses enhance growth and photosynthesis of the coral reef macroalga Sargassum baccularia. Marine Ecology Progress Series 170: 95-105.

Shevejko, S. (1982). Novaya krasnaya izvestkovaya vodorosl'. [A new red calcareous alga.]. Biologiya korallovykh rifov. Soobshchestva priavstralijskikh vod. [Biology of coral reefs. Associations in Australian nearshore waters.] [Russian.] Akademiya Nauk SSSR, Dal'nevostochnyj Nauchnyj Tsentr, Institut Biologii Morya: Vladivostok: 26-28.

The International Plant Names Index. (2011). Retrieved 2011, from http:/ / www.ipni.org.

Verheij, E. and Prud'homme van Reine, W. (1993). Seaweeds of the Spermonde Archipelago, SW Sulawesi, Indonesia. Blumea 37: 385-510.

Walker, D. and Prince, R. (1987). Distribution and biogeography of seagrass species on the northwest coast of Australia. Aquatic Botany 29: 19-32.
Walker, D.I. (1992). Part IX. Seagrasses (p. 75). In: Morgan, G.J. (ed.), Survey of the Aquatic Fauna of the Kimberley Islands and Reefs, Western Australia. Western Australian Museum: Perth. Unpublished report.

Walker, D.I. (1995). Part 5. Seagrasses and Macroalgae (pp. 58-66). Survey of the marine biota of the southern Kimberley Islands, Western Australia. Western Australian Museum: Perth. Unpublished report.

Walker, D.I. (1996). Part 5. Seagrasses and macroalgae (pp. 36-38). Survey of the marine biota of the eastern Kimberley, Western Australia. University of Western Australia, Western Australian Museum and Museum and Art Gallery of the Northern Territory. Unpublished report.

Walker, D. (1997). Part 5. Macroalgae and seagrasses (pp. 40-45). In: Walker, D.I. (ed.), Marine biological survey of the central Kimberley coast, Western Australia. University of Western Australia: Perth. Unpublished report.

Western Australian Herbarium. (2011). 'FloraBase-the Western Australian Flora.' Retrieved 2011, from http:/ / florabase.dec.wa.gov.au/

Womersley, H. (1984). The marine benthic flora of southern Australia, Part I. Introduction; collection and preservation; history of studies; ecology; biogeography; seagrasses (by E.L.Robertson); Chlorophyta and Charophyta. Government Printer: South Australia.

MANUSCRIPT RECEIVED 27 APRIL 2014; ACCEPTED 12 SEPTEMBER 2014. 